\title{
A New Ranking Principle For Ordering Trapezoidal Intuitionistic Fuzzy Numbers
}

\author{
Lakshmana Gomathi Nayagam Velu, Jeevaraj Selvaraj, and Dhanasekaran Ponnialagan \\ Department of Mathematics, National Institute of Technology, Tiruchirappalli, India \\ Correspondence should be addressed to Jeevaraj Selvaraj; alba.jeevi@gmail.com
}

Received 27 June 2016; Revised 4 November 2016; Accepted 13 November 2016; Published 6 February 2017

Academic Editor: Jose Egea

Copyright ( 2017 Lakshmana Gomathi Nayagam Velu et al. This is an open access article distributed under the Creative Commons Attribution License, which permits unrestricted use, distribution, and reproduction in any medium, provided the original work is properly cited.

\begin{abstract}
Modelling real life (industrial) problems using intuitionistic fuzzy numbers is inevitable in the present scenario due to their efficiency in solving problems and their accuracy in the results. Particularly, trapezoidal intuitionistic fuzzy numbers (TrIFNs) are widely used in describing impreciseness and incompleteness of a data. Any intuitionistic fuzzy decision-making problem requires the ranking procedure for intuitionistic fuzzy numbers. Ranking trapezoidal intuitionistic fuzzy numbers play an important role in problems involving incomplete and uncertain information. The available intuitionistic fuzzy decision-making methods cannot perform well in all types of problems, due to the partial ordering on the set of intuitionistic fuzzy numbers. In this paper, a new total ordering on the class of TrIFNs using eight different score functions, namely, imprecise score, nonvague score, incomplete score, accuracy score, spread score, nonaccuracy score, left area score, and right area score, is achieved and our proposed method is validated using illustrative examples. Significance of our proposed method with familiar existing methods is discussed.
\end{abstract}

\section{Introduction}

Classical set theory cannot be the better choice for modelling problems involving qualitative or imprecise information. To model such problems, fuzzy number was introduced by Jain [1] and some operations on fuzzy numbers are defined in $[2,3]$. Intuitionistic fuzzy numbers are comparatively better in modelling real life problems involving uncertainties and imprecise information. Particularly, trapezoidal intuitionistic fuzzy numbers are more effective in describing impreciseness and incompleteness of a data. To resolve the task of comparing trapezoidal intuitionistic fuzzy numbers, many authors have proposed different ranking methods but none of them yield a total order on the class of TrIFNs with finite number of scores. Different ranking methodologies on the class of intuitionistic fuzzy numbers are discussed in $[4,5]$.

Nehi and Maleki [6] generalised the idea of natural ordering on real numbers to the triangular intuitionistic fuzzy numbers (TIFNs) by adopting a statistical view point. Nehi [7] compared TIFNs using lexicographic technique. $\mathrm{Li}$ [2] developed the idea of value and ambiguity of a triangular intuitionistic fuzzy number and introduced the new ranking method using the concept of the ratio of the value index to the ambiguity index. Ye [8] presented the new ranking method using expected value of a trapezoidal intuitionistic fuzzy number and solved the decision-making problem using weighted expected value of TrIFN. Dubey and Mehara [9] extended the concept of value and ambiguity to the slightly modified TIFN and proposed a new approach to solve intuitionistic fuzzy linear programing problem. Nehi [7] introduced the concept of characteristic values of membership and nonmembership functions of TrIFN and proposed a new ranking method for trapezoidal intuitionistic fuzzy numbers by using it. Zhang and Nan [10] developed a compromise ratio ranking method for fuzzy multiattribute decision-making (MADM) problem based on the concept that larger TIFN among other TIFNs will be closer to the maximum value index and it will be far away from the minimum ambiguity index simultaneously. Kumar and Kaur [11] proposed the ranking method for TrIFNs by modifying Nehi's [10] method. Zeng et al. [12] introduced a new ranking method for TrIFNs by extending the concept value and ambiguity of TIFN defined in Li [2]. Wan and Dong [13] introduced the concept of lower and upper weighted 
possibility mean and possibility mean for a trapezoidal intuitionistic fuzzy numbers and proposed the new ranking method by use of it. Different ranking methods and their applications on multicriteria decision-making problem and other domains are studied in ([14-20]). Lakshmana Gomathi Nayagam et al. $[19,21,22]$ have introduced a complete ranking procedure on the class of intuitionistic fuzzy numbers using countable number of parameter. In this paper, a new total ordering on the class of TrIFNs using finite number of score functions is achieved. Also the limitations and drawbacks of all the abovementioned methods are discussed and the efficiency of our proposed method is shown by comparing all existing methods.

This paper is organised in the following manner. After introduction, some important definitions on intuitionistic fuzzy numbers are given in Section 2. The different subclasses of TrIFNs are introduced and the new score functions on these subclasses are established in Section 3. In Section 4, a complete ranking on the class of trapezoidal intuitionistic fuzzy numbers by using score functions defined in Section 3 is explained. The ranking procedure is explained in detail with several examples and also our proposed method is compared with some other existing methods in the Section 5. Finally conclusions are given in Section 6.

\section{Preliminaries}

Here we give a brief review of some preliminaries.

Definition 1 (Atanassov [23]). Let $X$ be a nonempty set. An intuitionistic fuzzy set (IFS) $A$ in $X$ is defined by $A=$ $\left\{\left\langle x, \mu_{A}(x), v_{A}(x)\right\rangle \mid x \in X\right\}$, where $\mu_{A}(x): X \rightarrow[0,1]$ and $v_{A}(x): X \rightarrow[0,1], x \in X$ with the conditions $0 \leq \mu_{A}(x)+$ $v_{A}(x) \leq 1, \forall x \in X$. The numbers $\mu_{A}(x), v_{A}(x) \in[0,1]$ denote the degree of membership and nonmembership of $x$ to lie in $A$, respectively. For each intuitionistic fuzzy subset $A$ in $X, \pi_{A}(x)=1-\mu_{A}(x)-\nu_{A}(x)$ is called hesitancy degree of $x$ to lie in $A$.

Definition 2 (Burillo et al., [24]). An intuitionistic fuzzy number $A=\left(\mu_{A}, v_{A}\right)$ in the set of real numbers $R$ is defined as

$$
\begin{aligned}
& \mu_{A}(x)= \begin{cases}f_{A}(x) & \text { if } a \leq x \leq b_{1} \\
1 & \text { if } b_{1} \leq x \leq b_{2} \\
g_{A}(x) & \text { if } b_{2} \leq x \leq c \\
0 & \text { Otherwise, }\end{cases} \\
& v_{A}(x)= \begin{cases}h_{A}(x) & \text { if } e \leq x \leq f_{1} \\
0 & \text { if } f_{1} \leq x \leq f_{2} \\
k_{A}(x) & \text { if } f_{2} \leq x \leq g \\
1 & \text { Otherwise, }\end{cases}
\end{aligned}
$$

where $0 \leq \mu_{A}(x)+v_{A}(x) \leq 1$ and $a, b_{1}, b_{2}, c, e, f_{1}, f_{2}, g \in \Re$ such that $e \leq a, f_{1} \leq b_{1} \leq b_{2} \leq f_{2}, c \leq g$, and four functions $f_{A}, g_{A}, h_{A}, k_{A}: \mathfrak{R} \rightarrow[0,1]$ are the legs of membership

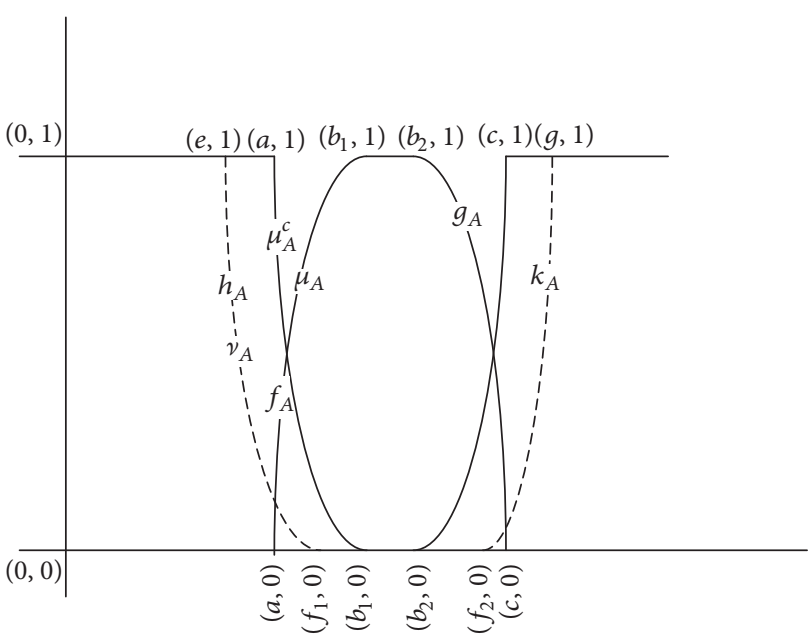

FIGURe 1: Intuitionistic fuzzy number.

function $\mu_{A}$ and nonmembership function $\nu_{A}$. The functions $f_{A}$ and $k_{A}$ are nondecreasing continuous functions and the functions $h_{A}$ and $g_{A}$ are nonincreasing continuous functions.

An intuitionistic fuzzy number $\left\{\left(a, b_{1}, b_{2}, c\right),\left(e, f_{1}, f_{2}, g\right)\right\}$ with $\left(e, f_{1}, f_{2}, g\right) \leq\left(a, b_{1}, b_{2}, c\right)^{c}$ is shown in Figure 1 .

Definition 3. A trapezoidal intuitionistic fuzzy number $A$ with parameters $e \leq a, f_{1} \leq b_{1} \leq b_{2} \leq f_{2}, c \leq g$ is denoted as $A=\left\{\left(a, b_{1}, b_{2}, c\right),\left(e, f_{1}, f_{2}, g\right)\right\}$ in the set of real numbers $\mathfrak{R}$ is an intuitionistic fuzzy number whose membership function and nonmembership function are given as

$$
\begin{aligned}
& \mu_{A}(x)= \begin{cases}\frac{x-a_{1}}{a_{2}-a_{1}} & \text { if } a_{1} \leq x \leq a_{2} \\
1 & \text { if } a_{2} \leq x \leq a_{3} \\
\frac{a_{4}-x}{a_{4}-a_{3}} & \text { if } a_{3} \leq x \leq a_{4} \\
0 & \text { Otherwise, }\end{cases} \\
& v_{A}(x)= \begin{cases}\frac{x-c_{2}}{c_{1}-c_{2}} & \text { if } c_{1} \leq x \leq c_{2} \\
0 & \text { if } c_{2} \leq x \leq c_{3} \\
\frac{x-c_{3}}{c_{4}-c_{3}} & \text { if } c_{3} \leq x \leq c_{4} \\
1 & \text { Otherwise. }\end{cases}
\end{aligned}
$$

If $a_{2}=a_{3}$ (and $c_{2}=c_{3}$ ) in a trapezoidal intuitionistic fuzzy number $A$, we have the triangular intuitionistic fuzzy numbers as special case of the trapezoidal intuitionistic fuzzy numbers.

A trapezoidal intuitionistic fuzzy number $A=\left\{\left(a, b_{1}\right.\right.$, $\left.\left.b_{2}, c\right),\left(e, f_{1}, f_{2}, g\right)\right\}$ with $f_{1} \leq b_{1}, f_{2} \geq b_{2}, e \leq a$, and $g \geq c$ is shown in Figure 2.

We note that the condition $\left(e, f_{1}, f_{2}, g\right) \leq\left(a, b_{1}, b_{2}, c\right)^{c}$ of the trapezoidal intuitionistic fuzzy number $A=\left\{\left(a, b_{1}\right.\right.$, $\left.\left.b_{2}, c\right),\left(e, f_{1}, f_{2}, g\right)\right\}$ whose membership and nonmembership fuzzy numbers of $A$ are $\left(a, b_{1}, b_{2}, c\right)$ and $\left(e, f_{1}, f_{2}, g\right)$ implies 


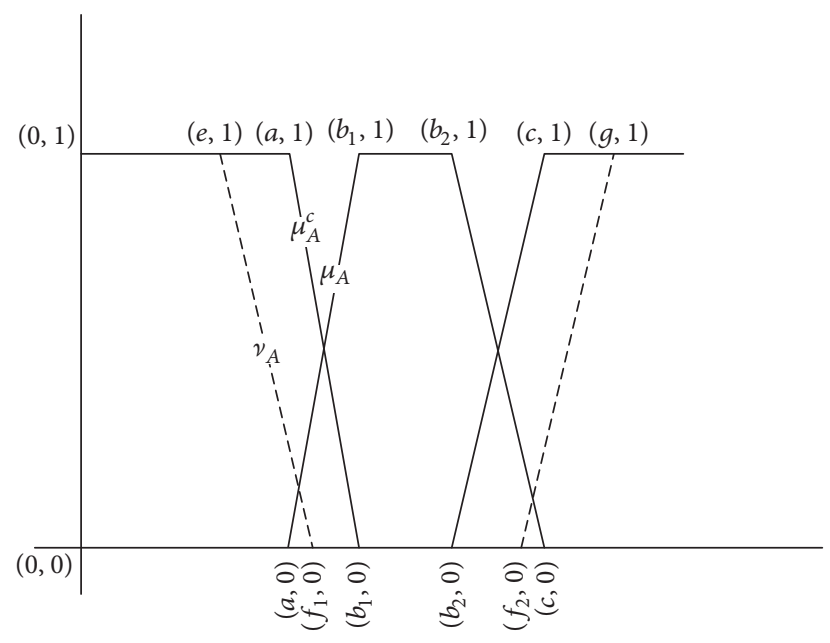

Figure 2: Trapezoidal intuitionistic fuzzy number.

$f_{1} \leq b_{1}, f_{2} \geq b_{2}, e \leq a$, and $g \geq c$ on the legs of trapezoidal intuitionistic fuzzy number.

Definition 4 (Atanassov and Gargov, [25]). Let $D[0,1]$ be the set of all closed subintervals of the interval $[0,1]$. An interval valued intuitionistic fuzzy set on a set $X \neq \phi$ is an expression given by $A=\left\{\left\langle x, \mu_{A}(x), v_{A}(x)\right\rangle: x \in X\right\}$, where $\mu_{A}: X \rightarrow$ $D[0,1], v_{A}: X \rightarrow D[0,1]$ with the condition $0<\sup _{x} \mu_{A}(x)+$ $\sup _{x} v_{A}(x) \leq 1$.

The intervals $\mu_{A}(x)$ and $\nu_{A}(x)$ denote, respectively, the degree of belongingness and nonbelongingness of the element $x$ to the set $A$. Thus for each $x \in X, \mu_{A}(x)$ and $\nu_{A}(x)$ are closed intervals whose lower and upper end points are, respectively, denoted by $\mu_{A_{L}}(x), \mu_{A_{U}}(x)$ and $\nu_{A_{L}}(x), \mu_{A_{U}}(x)$. We denote $A=\left\{\left\langle x,\left[\mu_{A_{L}}(x), \mu_{A_{U}}(x)\right],\left[\nu_{A_{L}}(x), \nu_{A_{U}}(x)\right]\right\rangle: x \in\right.$ $X\}$, where $0<\mu_{A}(x)+\nu_{A}(x) \leq 1$.

For each element $x \in X$, we can compute the unknown degree (hesitance degree) of belongingness $\pi_{A}(x)$ to $A$ as $\pi_{A}(x)=1-\mu_{A}(x)-v_{A}(x)=\left[1-\mu_{A_{U}}(x)-v_{A_{U}}(x), 1-\right.$ $\left.\mu_{A_{L}}(x)-v_{A_{L}}(x)\right]$. An intuitionistic fuzzy interval number (IFIN) is denoted by $A=([a, b],[c, d])$ for convenience.

Definition 5. Let $\alpha, \beta \in(0,1]$. An $(\alpha, \beta)$-cut of a trapezoidal intuitionistic fuzzy number, denoted by ${ }^{(\alpha, \beta)} A_{I}$, is defined as ${ }^{(\alpha, \beta)} A_{I}=\left\{\left({ }^{\alpha} \mu_{A_{I}},{ }^{\beta} v_{A_{I}}\right)\right\}$, where ${ }^{\alpha} \mu_{A_{I}}$ and ${ }^{\beta} \nu_{A_{I}}$ are $\alpha$-cut and $\beta$-cut of membership and nonmembership trapezoidal fuzzy numbers.

The $(\alpha, \beta)$-cut of trapezoidal intuitionistic fuzzy number $A_{I}=\left\langle\left(a_{1 \mu}, a_{2 \mu}, a_{3 \mu}, a_{4 \mu}\right),\left(a_{1 v}^{\prime}, a_{2 v}^{\prime}, a_{3 v}^{\prime}, a_{4 v}^{\prime}\right)\right\rangle$ is given by ${ }^{(\alpha, \beta)} A_{I}=\left\{\left[{ }^{\alpha} \mu_{A_{I_{L}}},{ }^{\alpha} \mu_{A_{I_{U}}}\right],\left[{ }^{\beta} v_{A_{I_{L}}},{ }^{\beta} v_{A_{I_{U}}}\right]\right\}=\left(\left[a_{1 \mu}+\alpha\left(a_{2 \mu}-\right.\right.\right.$ $\left.\left.\left.a_{1 \mu}\right), a_{4 \mu}-\alpha\left(a_{4 \mu}-a_{3 \mu}\right)\right],\left[a_{1 v}^{\prime}+\beta\left(a_{2 v}^{\prime}-a_{1 v}^{\prime}\right), a_{4 \nu}^{\prime}-\beta\left(a_{4 v}^{\prime}-a_{3 \nu}^{\prime}\right)\right]\right)$, where ${ }^{\alpha} \mu_{A_{I_{L}}}$ and ${ }^{\alpha} \mu_{A_{I_{U}}}$ represents the lower and upper end points of the $\alpha$-cut of the membership function of $A_{I}$ and ${ }^{\beta} v_{A_{I_{L}}}$ and ${ }^{\beta} v_{A_{I_{U}}}$ represent the lower and upper end points of the $\beta$-cut of the nonmembership function of $A_{I}$, respectively.

\section{Different Classes of Trapezoidal Intuitionistic Fuzzy Numbers}

In this section the entire class of TrIFNs is partitioned into eight subclasses and further score functions using different concepts are defined in order to give total order on the entire class of TrIFNs and some theorems related to these concepts are established.

In this paper, $A_{I}$ and $B_{I}$ always denote trapezoidal intuitionistic fuzzy number (TrIFN).

$A_{I}=\left\langle\left(a_{1 \mu}, a_{2 \mu}, a_{3 \mu}, a_{4 \mu}\right),\left(a_{1 v}^{\prime}, a_{2 v}^{\prime}, a_{3 v}^{\prime}, a_{4 v}^{\prime}\right)\right\rangle$ with conditions that $a_{1 \nu}^{\prime} \leq a_{1 \mu}$ and $a_{2 \nu}^{\prime} \leq a_{2 \mu}, a_{3 \nu}^{\prime} \geq a_{3 \mu}$ and $a_{4 \nu}^{\prime} \geq a_{4 \mu}$ and $B_{I}=\left\langle\left(b_{1 \mu}, b_{2 \mu}, b_{3 \mu}, b_{4 \mu}\right),\left(b_{1 v}^{\prime}, b_{2 v}^{\prime}, b_{3 v}^{\prime}, b_{4 v}^{\prime}\right)\right\rangle$ with the condition that $b_{1 v}^{\prime} \leq b_{1 \mu}$ and $b_{2 v}^{\prime} \leq b_{2 \mu}, b_{3 v}^{\prime} \geq b_{3 \mu}$, and $b_{4 v}^{\prime} \geq b_{4 \mu}$ unless otherwise specifically stated.

3.1. Imprecise Score of a Trapezoidal Intuitionistic Fuzzy Number. In this subsection, a new subclass of TrIFNs is introduced. The imprecise score function on this class of trapezoidal intuitionistic fuzzy numbers is defined and some of its properties are studied using illustrative examples.

Definition 6. Let $A_{I}=\left\langle\left(a_{1 \mu}, a_{2 \mu}, a_{3 \mu}, a_{4 \mu}\right),\left(a_{1 v}^{\prime}, a_{2 v}^{\prime}, a_{3 v}^{\prime}, a_{4 v}^{\prime}\right)\right\rangle$ and $B_{I}=\left\langle\left(b_{1 \mu}, b_{2 \mu}, b_{3 \mu}, b_{4 \mu}\right),\left(b_{1 v}^{\prime}, b_{2 v}^{\prime}, b_{3 v}^{\prime}, b_{4 v}^{\prime}\right)\right\rangle$ be two TrIFNs.

A special subclass $C_{1}$ of the set of TrIFNs consist of TrIFNs for which every pair of $A_{I}$ and $B_{I}$ are with $a_{1 \mu} \geq b_{1 \mu}, a_{2 \mu} \geq$ $b_{2 \mu}, a_{3 \mu} \leq b_{3 \mu}, a_{4 \mu} \leq b_{4 \mu}$ and $a_{1 v}^{\prime} \geq b_{1 v}^{\prime}, a_{2 v}^{\prime} \geq b_{2 v}^{\prime}, a_{3 \nu}^{\prime} \leq$ $b_{3 v}^{\prime}, a_{4 v}^{\prime} \leq b_{4 v}^{\prime}$. The imprecise relation on $C_{1}$ is denoted as $\sqsubseteq$ and defined as follows: if $A_{I}, B_{I} \in C_{1} \subset$ TrIFN such that $A_{I} \sqsubseteq B_{I}$ then $a_{1 \mu} \geq b_{1 \mu}, a_{2 \mu} \geq b_{2 \mu}, a_{3 \mu} \leq b_{3 \mu}, a_{4 \mu} \leq b_{4 \mu}$ and $a_{1 v}^{\prime} \geq b_{1 v}^{\prime}, a_{2 v}^{\prime} \geq b_{2 v}^{\prime}, a_{3 v}^{\prime} \leq b_{3 v}^{\prime}, a_{4 v}^{\prime} \leq b_{4 v}^{\prime}$.

If $A_{I} \sqsubset B_{I}$ then one of the above inequalities becomes strict inequality.

Note 1. By Definition 6, we note that any pair of members of $C_{1}$ are related under .

The score function which measures the preciseness is defined as follows.

Definition 7. Let $A_{I}=\left\langle\left(a_{1 \mu}, a_{2 \mu}, a_{3 \mu}, a_{4 \mu}\right),\left(a_{1 v}^{\prime}, a_{2 v}^{\prime}, a_{3 v}^{\prime}, a_{4 v}^{\prime}\right)\right\rangle$ be a trapezoidal intuitionistic fuzzy number with $a_{1 \mu}$, $a_{2 \mu}, a_{3 \mu}, a_{4 \mu}, a_{1 \nu}^{\prime}, a_{2 v}^{\prime}, a_{3 v}^{\prime}, a_{4 \nu}^{\prime} \in[0,1]$. Then the imprecise score of a trapezoidal intuitionistic fuzzy number $A_{I}$ is defined by

$$
\begin{aligned}
& J_{1}\left(A_{I}\right) \\
& =\int_{0}^{1} \frac{\left[{ }^{\alpha} \mu_{A_{I_{L}}}-{ }^{\alpha} \mu_{A_{I_{U}}}\right]}{4} d \alpha+\int_{0}^{1} \frac{\left[\beta \nu_{A_{I_{L}}}-{ }^{\beta} \nu_{A_{I_{U}}}\right]}{4} d \beta \\
& =\int_{0}^{1} \frac{\left(a_{1 \mu}+\alpha\left(a_{2 \mu}-a_{1 \mu}\right)\right)}{4} d \alpha \\
& \quad-\int_{0}^{1} \frac{\left(a_{4 \mu}-\alpha\left(a_{4 \mu}-a_{3 \mu}\right)\right)}{4} d \alpha
\end{aligned}
$$




$$
\begin{aligned}
& +\int_{0}^{1} \frac{\left(a_{1 \nu}^{\prime}+\beta\left(a_{2 v}^{\prime}-a_{1 \nu}^{\prime}\right)\right)}{4} d \beta \\
& -\int_{0}^{1} \frac{\left(a_{4 v}^{\prime}-\beta\left(a_{4 v}^{\prime}-a_{3 \nu}^{\prime}\right)\right)}{4} d \beta \\
& =\frac{\left(a_{1 \mu}+a_{2 \mu}\right)-\left(a_{3 \mu}+a_{4 \mu}\right)+\left(a_{1 \nu}^{\prime}+a_{2 \nu}^{\prime}\right)-\left(a_{3 \nu}^{\prime}+a_{4 v}^{\prime}\right)}{8} .
\end{aligned}
$$

The proofs of the following propositions are immediate from the above definition and hence they are omitted.

Proposition 8. For any real number $r \in[0,1], J_{1}(r)=0$.

Proposition 9. If $A_{I}=\left(a_{1 \mu}, a_{2 \mu}, a_{3 \mu}, a_{4 \mu}\right)$ is a trapezoidal fuzzy number, then $J_{1}\left(A_{I}\right)=\left(\left(a_{1 \mu}+a_{2 \mu}\right)-\left(a_{3 \mu}+a_{4 \mu}\right)\right) / 4$.

Proposition 10. Let $A_{I}=\left\langle\left(a_{1 \mu}, a_{2 \mu}, a_{3 \mu}\right),\left(a_{1 \nu}^{\prime}, a_{2 v}^{\prime}, a_{3 \nu}^{\prime}\right)\right\rangle$ be a triangular intuitionistic fuzzy number. Then $J_{1}\left(A_{I}\right)=\left(\left(a_{1 \mu}-\right.\right.$ $\left.\left.a_{3 \mu}\right)+\left(a_{1 v}^{\prime}-a_{3 v}^{\prime}\right)\right) / 4$.

Proposition 11. Let $A_{I}=\left(\left[a_{1 \mu}, a_{2 \mu}\right],\left[a_{1 \nu}^{\prime}, a_{2 \gamma}^{\prime}\right]\right)$ be an interval valued intuitionistic fuzzy number. Then $J_{1}\left(A_{I}\right)=\left(\left(a_{1 \mu}-a_{2 \mu}\right)+\right.$ $\left.\left(a_{1 v}^{\prime}-a_{2 v}^{\prime}\right)\right) / 2$.

Theorem 12. Let $A_{I}$ and $B_{I} \in C_{1}$. If $A_{I} \sqsubset B_{I}$ then $J_{1}\left(A_{I}\right)>$ $J_{1}\left(B_{I}\right)$.

Proof. Let $A_{I} \sqsubset B_{I}$. We claim that $J_{1}\left(A_{I}\right)-J_{1}\left(B_{I}\right)>0$. By Definition 6, we have

$$
\begin{aligned}
& a_{1 \mu} \geq b_{1 \mu}, \\
& a_{2 \mu} \geq b_{2 \mu}, \\
& a_{3 \mu} \leq b_{3 \mu}, \\
& a_{4 \mu} \leq b_{4 \mu}, \\
& a_{1 v}^{\prime} \geq b_{1 v}^{\prime}, \\
& a_{2 v}^{\prime} \geq b_{2 v}^{\prime}, \\
& a_{3 v}^{\prime} \leq b_{3 v}^{\prime}, \\
& a_{4 v}^{\prime} \leq b_{4 v}^{\prime} .
\end{aligned}
$$

Now,

$$
\begin{gathered}
8\left(J_{1}\left(A_{I}\right)-J_{1}\left(B_{I}\right)\right)=\left[\left(a_{1 \mu}-b_{1 \mu}\right)+\left(a_{2 \mu}-b_{2 \mu}\right)\right. \\
\left.+\left(b_{3 \mu}-a_{3 \mu}\right)+\left(b_{4 \mu}-a_{4 \mu}\right)\right]+\left[\left(a_{1 \nu}^{\prime}-b_{1 \nu}^{\prime}\right)\right. \\
\left.+\left(a_{2 v}^{\prime}-b_{2 v}^{\prime}\right)+\left(b_{3 \nu}^{\prime}-a_{3 \nu}^{\prime}\right)+\left(b_{4 v}^{\prime}-a_{4 v}^{\prime}\right)\right] .
\end{gathered}
$$

From (4) it is very easy to see that all the terms in (5) are positive. Therefore their sum is also positive. From Definition 6, we know that at least one of the above inequalities in (4) becomes strict inequality and hence we get $J_{1}\left(A_{I}\right)-J_{1}\left(B_{I}\right)>$ 0 .
Theorem 13. Let $A_{I}$ and $B_{I} \in C_{1}$ such that $J_{1}\left(A_{I}\right)=J_{1}\left(B_{I}\right)$; then $A_{I}=B_{I}$.

Proof. Let $J_{1}\left(A_{I}\right)=J_{1}\left(B_{I}\right)$. We claim that $A_{I}=B_{I}$. By Definition 6, without loss of generality, we have

$$
\begin{aligned}
& a_{1 \mu} \geq b_{1 \mu}, \\
& a_{2 \mu} \geq b_{2 \mu}, \\
& a_{3 \mu} \leq b_{3 \mu}, \\
& a_{4 \mu} \leq b_{4 \mu}, \\
& a_{1 v}^{\prime} \geq b_{1 v}^{\prime}, \\
& a_{2 v}^{\prime} \geq b_{2 v}^{\prime}, \\
& a_{3 v}^{\prime} \leq b_{3 v}^{\prime}, \\
& a_{4 v}^{\prime} \leq b_{4 v}^{\prime} .
\end{aligned}
$$

Now,

$$
\begin{gathered}
8\left(J_{1}\left(A_{I}\right)-J_{1}\left(B_{I}\right)\right)=\left[\left(a_{1 \mu}-b_{1 \mu}\right)+\left(a_{2 \mu}-b_{2 \mu}\right)\right. \\
\left.+\left(b_{3 \mu}-a_{3 \mu}\right)+\left(b_{4 \mu}-a_{4 \mu}\right)\right]+\left[\left(a_{1 \nu}^{\prime}-b_{1 \nu}^{\prime}\right)\right. \\
\left.+\left(a_{2 \nu}^{\prime}-b_{2 \nu}^{\prime}\right)+\left(b_{3 \nu}^{\prime}-a_{3 \nu}^{\prime}\right)+\left(b_{4 v}^{\prime}-a_{4 \nu}^{\prime}\right)\right]=0 .
\end{gathered}
$$

Therefore from (6) and (7), it is clear that all the terms in (7) are positive and their sum gives zero only when each term is equal to zero. Hence $a_{1 \mu}=b_{1 \mu}, a_{2 \mu}=b_{2 \mu}, a_{3 \mu}=b_{3 \mu}, a_{4 \mu}=$ $b_{4 \mu}$ and $a_{1 v}^{\prime}=b_{1 \nu}^{\prime}, a_{2 v}^{\prime}=b_{2 v}^{\prime}, a_{3 \nu}^{\prime}=b_{3 \nu}^{\prime}, a_{4 v}^{\prime}=b_{4 v}^{\prime}$, hence the proof.

Note 2. The imprecise score can be calculated to any TrIFN but $J_{1}$ gives total order in $C_{1}$.

Definition 14. If $J_{1}\left(A_{I}\right)>J_{1}\left(B_{I}\right)\left(J_{1}\left(A_{I}\right)<J_{1}\left(B_{I}\right)\right)$, then $A_{I}>$ $B_{I}\left(A_{I}<B_{I}\right)$.

The following example explains the ranking procedure introduced in Definition 14.

Example 15. Let $A_{I}=\langle(0.1,0.2,0.35,0.5),(0,0.15,0.45,0.6)\rangle$ and $B_{I}=\langle(0.15,0.2,0.35,0.45),(0.1,0.1,0.5,0.55)\rangle \in C_{1}$. Now $J_{1}\left(A_{I}\right)=-0.18125$ and $J_{1}\left(B_{I}\right)=-0.1625, J_{1}\left(A_{I}\right)<$ $J_{1}\left(B_{I}\right)$.

Hence $A_{I}<B_{I}$.

Example 16. Let $A_{I}=\left\langle\left(a_{1 \mu}, a_{2 \mu}, a_{3 \mu}, a_{4 \mu}\right),\left(a_{1 v}^{\prime}, a_{2 v}^{\prime}, a_{3 v}^{\prime}, a_{4 v}^{\prime}\right)\right\rangle$ and $B_{I}=\left\langle\left(a_{1 \mu}+\epsilon, a_{2 \mu}+\epsilon, a_{3 \mu}-\epsilon, a_{4 \mu}-\epsilon\right),\left(a_{1 v}^{\prime}-\epsilon, a_{2 v}^{\prime}-\right.\right.$ $\left.\left.\epsilon, a_{3 v}^{\prime}+\epsilon, a_{4 v}^{\prime}+\epsilon\right)\right\rangle \in \operatorname{TrIFN}$ but not in $C_{1}$, where $\epsilon \in[0,1]$.

Now $J_{1}\left(A_{I}\right)=J_{1}\left(B_{I}\right)=\left(\left(a_{1 \mu}+a_{2 \mu}\right)-\left(a_{3 \mu}+a_{4 \mu}\right)+\left(a_{1 \nu}^{\prime}+\right.\right.$ $\left.\left.a_{2 v}^{\prime}\right)-\left(a_{3 v}^{\prime}+a_{4 v}^{\prime}\right)\right) / 8$. But $A_{I} \neq B_{I}$. Pictorial representation of this example is given in Figure 3.

Example 16 shows that $J_{1}$ is not enough cover the entire class of TrIFNs. Therefore it is needed for us to define another 


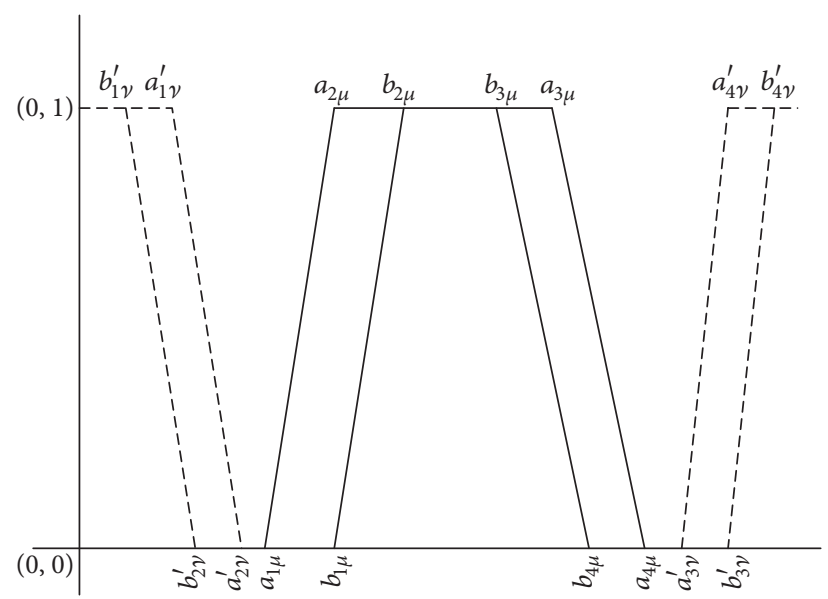

FIgURE 3: Pictorial representation of Example 16.

score function that can cover some subclass of TrIFNs which cannot be covered by $J_{1}$.

3.2. Nonvague Score of a Trapezoidal Intuitionistic Fuzzy Number. In this subsection, another subclass of TrIFNs is introduced using nonvague relation. The nonvague score function on this class of trapezoidal intuitionistic fuzzy numbers is defined and some of its properties are studied using illustrative examples.

Definition 17. Let $A_{I}$ and $B_{I}$ be two TrIFNs.

A special subclass $C_{2}$ of the set of TrIFNs consist of TrIFNs for which every pair of $A_{I}$ and $B_{I}$ are with $a_{1 \mu} \geq b_{1 \mu}, a_{2 \mu} \geq$ $b_{2 \mu}, a_{3 \mu} \leq b_{3 \mu}, a_{4 \mu} \leq b_{4 \mu}$ and $a_{1 v}^{\prime} \leq b_{1 v}^{\prime}, a_{2 v}^{\prime} \leq b_{2 v}^{\prime}, a_{3 v}^{\prime} \geq$ $b_{3 v}^{\prime}, a_{4 v}^{\prime} \geq b_{4 v}^{\prime}$. The nonvague relation on $C_{2}$ is denoted as $\sqsupseteq$ and defined as follows: if $A_{I}, B_{I} \in C_{2} \subset$ TrIFN such that $A_{I} \sqsupseteq B_{I}$ then $a_{1 \mu} \geq b_{1 \mu}, a_{2 \mu} \geq b_{2 \mu}, a_{3 \mu} \leq b_{3 \mu}, a_{4 \mu} \leq b_{4 \mu}$ and $a_{1 v}^{\prime} \leq b_{1 v}^{\prime}, a_{2 v}^{\prime} \leq b_{2 v}^{\prime}, a_{3 v}^{\prime} \geq b_{3 v}^{\prime}, a_{4 v}^{\prime} \geq b_{4 v}^{\prime}$.

If $A_{I} \sqsupset B_{I}$ then one of the above inequalities becomes strict inequality.

Note 3. By Definition 17, we note that any pair of members of $\mathrm{C}_{2}$ are related under $\lrcorner$.

The score function which measures the nonvagueness is defined as follows.

Definition 18. Let $A_{I}$ be a trapezoidal intuitionistic fuzzy number. Then the nonvague score of a trapezoidal intuitionistic fuzzy number $A_{I}$ is defined by

$$
\begin{aligned}
& J_{2}\left(A_{I}\right) \\
& =\int_{0}^{1} \frac{\left[{ }^{\alpha} \mu_{A_{I_{L}}}-{ }^{\alpha} \mu_{A_{I_{U}}}\right]}{4} d \alpha-\int_{0}^{1} \frac{\left[{ }^{\beta}{ }_{A_{I_{L}}}-{ }^{\beta} v_{A_{I_{U}}}\right]}{4} d \beta \\
& =\frac{\left(a_{1 \mu}+a_{2 \mu}\right)-\left(a_{3 \mu}+a_{4 \mu}\right)-\left(a_{1 v}^{\prime}+a_{2 v}^{\prime}\right)+\left(a_{3 \nu}^{\prime}+a_{4 v}^{\prime}\right)}{8} .
\end{aligned}
$$

The proofs of the following propositions are immediate from Definition 18 and hence they are omitted.

Proposition 19. For any real number $r \in[0,1], J_{2}(r)=0$.

Proposition 20. If $A_{I}=\left(a_{1 \mu}, a_{2 \mu}, a_{3 \mu}, a_{4 \mu}\right)$ is a trapezoidal fuzzy number, then $J_{2}\left(A_{I}\right)=0$.

Proposition 21. Let $A_{I}=\left\langle\left(a_{1 \mu}, a_{2 \mu}, a_{3 \mu}\right),\left(a_{1 v}^{\prime}, a_{2 v}^{\prime}, a_{3 v}^{\prime}\right)\right\rangle$ be a triangular intuitionistic fuzzy number. Then $J_{2}\left(A_{I}\right)=\left(\left(a_{1 \mu}-\right.\right.$ $\left.\left.a_{3 \mu}\right)+\left(-a_{1 v}^{\prime}+a_{3 \nu}^{\prime}\right)\right) / 8$.

Proposition 22. Let $A_{I}=\left(\left[a_{1 \mu}, a_{2 \mu}\right],\left[a_{1 v}^{\prime}, a_{2 v}^{\prime}\right]\right)$ be an interval valued intuitionistic fuzzy number. Then $J_{2}\left(A_{I}\right)=\left(\left(a_{1 \mu}-a_{2 \mu}\right)+\right.$ $\left.\left(-a_{1 v}^{\prime}+a_{2 v}^{\prime}\right)\right) / 4$.

Theorem 23. Let $A_{I}$ and $B_{I} \in C_{2}$. If $A_{I} \sqsupset B_{I}$ then $J_{2}\left(A_{I}\right)>$ $J_{2}\left(B_{I}\right)$.

Proof. Let us assume $A_{I} \sqsupset B_{I}$. We claim that $J_{2}\left(A_{I}\right)-J_{2}\left(B_{I}\right)>$ 0 .

By Definition 17, we have

$$
\begin{aligned}
& a_{1 \mu} \geq b_{1 \mu}, \\
& a_{2 \mu} \geq b_{2 \mu}, \\
& a_{3 \mu} \leq b_{3 \mu}, \\
& a_{4 \mu} \leq b_{4 \mu}, \\
& a_{1 v}^{\prime} \leq b_{1 v}^{\prime}, \\
& a_{2 v}^{\prime} \leq b_{2 v}^{\prime}, \\
& a_{3 v}^{\prime} \geq b_{3 v}^{\prime}, \\
& a_{4 v}^{\prime} \geq b_{4 v}^{\prime} .
\end{aligned}
$$

Now,

$$
\begin{gathered}
8\left(J_{2}\left(A_{I}\right)-J_{2}\left(B_{I}\right)\right)=\left[\left(a_{1 \mu}-b_{1 \mu}\right)+\left(a_{2 \mu}-b_{2 \mu}\right)\right. \\
\left.+\left(b_{3 \mu}-a_{3 \mu}\right)+\left(b_{4 \mu}-a_{4 \mu}\right)\right]+\left[\left(b_{1 v}^{\prime}-a_{1 v}^{\prime}\right)\right. \\
\left.+\left(b_{2 v}^{\prime}-a_{2 v}^{\prime}\right)+\left(a_{3 \nu}^{\prime}-b_{3 v}^{\prime}\right)+\left(a_{4 v}^{\prime}-b_{4 v}^{\prime}\right)\right] .
\end{gathered}
$$

From (9) it is very easy to see that all the terms in (10) are positive. Therefore their sum is also positive. From Definition 17, we know that at least one of the above inequalities in (9) becomes strict inequality and hence we get $J_{2}\left(A_{I}\right)-J_{2}\left(B_{I}\right)>$ 0 .

Theorem 24. Let $A_{I}$ and $B_{I} \in C_{2}$. If $J_{2}\left(A_{I}\right)=J_{2}\left(B_{I}\right)$ then $A_{I}=B_{I}$.

Proof. Let us assume $J_{2}\left(A_{I}\right)=J_{2}\left(B_{I}\right)$. We claim that $A_{I}=B_{I}$. 
By Definition 17, without loss of generality, we have

$$
\begin{aligned}
& a_{1 \mu} \geq b_{1 \mu}, \\
& a_{2 \mu} \geq b_{2 \mu}, \\
& a_{3 \mu} \leq b_{3 \mu}, \\
& a_{4 \mu} \leq b_{4 \mu}, \\
& a_{1 v}^{\prime} \leq b_{1 v}^{\prime}, \\
& a_{2 v}^{\prime} \leq b_{2 v}^{\prime}, \\
& a_{3 v}^{\prime} \geq b_{3 v}^{\prime}, \\
& a_{4 v}^{\prime} \geq b_{4 v}^{\prime} .
\end{aligned}
$$

Now,

$$
\begin{gathered}
8\left(J_{2}\left(A_{I}\right)-J_{2}\left(B_{I}\right)\right)=\left[\left(a_{1 \mu}-b_{1 \mu}\right)+\left(a_{2 \mu}-b_{2 \mu}\right)\right. \\
\left.+\left(b_{3 \mu}-a_{3 \mu}\right)+\left(b_{4 \mu}-a_{4 \mu}\right)\right]+\left[\left(b_{1 \nu}^{\prime}-a_{1 \nu}^{\prime}\right)\right. \\
\left.+\left(b_{2 \nu}^{\prime}-a_{2 \nu}^{\prime}\right)+\left(a_{3 \nu}^{\prime}-b_{3 \nu}^{\prime}\right)+\left(a_{4 \nu}^{\prime}-b_{4 \nu}^{\prime}\right)\right]=0 .
\end{gathered}
$$

Therefore from (11) and (12), it is clear that all the terms in (12) are positive; therefore their sum gives zero only when each term is equal to zero. Hence $a_{1 \mu}=b_{1 \mu}, a_{2 \mu}=b_{2 \mu}, a_{3 \mu}=$ $b_{3 \mu}, a_{4 \mu}=b_{4 \mu}$ and $a_{1 v}^{\prime}=b_{1 v}^{\prime}, a_{2 v}^{\prime}=b_{2 v}^{\prime}, a_{3 v}^{\prime}=b_{3 v}^{\prime}, a_{4 v}^{\prime}=b_{4 v}^{\prime}$. Hence $A_{I}=B_{I}$.

Note 4. The nonvague score can be calculated to any TrIFN. But $J_{2}$ gives total order on $C_{2}$.

Definition 25. If $J_{2}\left(A_{I}\right)>J_{2}\left(B_{I}\right)\left(J_{2}\left(A_{I}\right)<J_{2}\left(B_{I}\right)\right)$, then $A_{I}>$ $B_{I}\left(A_{I}<B_{I}\right)$.

Ranking relation defined above is explained in the following example.

Example 26. Let $A_{I}=\langle(0.15,0.25,0.35,0.45),(0.1,0.2,0.4$, $0.5)\rangle$ and $B_{I}=\langle(0.18,0.25,0.35,0.4),(0,0.15,0.45,0.55)\rangle \epsilon$ $C_{2}$. Now $J_{2}\left(A_{I}\right)=0.025$ and $J_{2}\left(B_{I}\right)=0.06625, J_{2}\left(B_{I}\right)>$ $J_{2}\left(A_{I}\right)$.

Hence $B_{I}>A_{I}$.

Example 27 shows the inefficiency of $J_{1}$ in comparing any two arbitrary TrIFNs and the importance of defining new score function $J_{2}$.

Example 27. Let $A_{I}=\left\langle\left(a_{1 \mu}, a_{2 \mu}, a_{3 \mu}, a_{4 \mu}\right),\left(a_{1 v}^{\prime}, a_{2 v}^{\prime}, a_{3 v}^{\prime}, a_{4 v}^{\prime}\right)\right\rangle$ and $B_{I}=\left\langle\left(a_{1 \mu}+\epsilon, a_{2 \mu}+\epsilon, a_{3 \mu}-\epsilon, a_{4 \mu}-\epsilon\right),\left(a_{1 v}^{\prime}-\epsilon, a_{2 v}^{\prime}-\right.\right.$ $\left.\left.\epsilon, a_{3 \nu}^{\prime}+\epsilon, a_{4 v}^{\prime}+\epsilon\right)\right\rangle \in \operatorname{TrIFN}$ and $\epsilon \in[0,1]$.

Now

$$
\begin{aligned}
& J_{1}\left(A_{I}\right)=J_{1}\left(B_{I}\right) \\
& =\frac{\left(a_{1 \mu}+a_{2 \mu}\right)-\left(a_{3 \mu}+a_{4 \mu}\right)+\left(a_{1 \nu}^{\prime}+a_{2 v}^{\prime}\right)-\left(a_{3 \nu}^{\prime}+a_{4 \nu}^{\prime}\right)}{8} \Longrightarrow
\end{aligned}
$$

$A_{I}=B_{I}$.

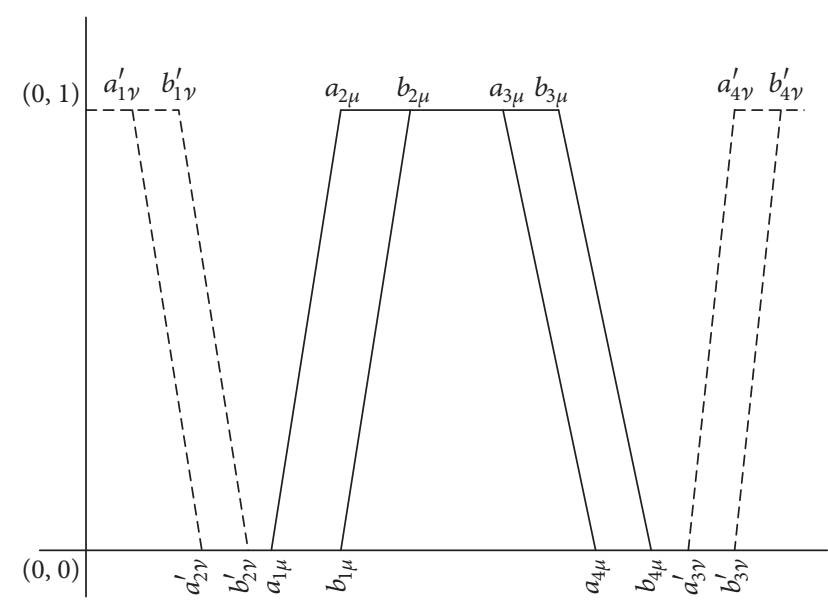

FIgURE 4: Pictorial representation of Example 28.

But

$$
\begin{aligned}
& J_{2}\left(A_{I}\right) \\
& =\frac{\left(a_{1 \mu}+a_{2 \mu}\right)-\left(a_{3 \mu}+a_{4 \mu}\right)-\left(a_{1 \nu}^{\prime}+a_{2 \nu}^{\prime}\right)+\left(a_{3 \nu}^{\prime}+a_{4 v}^{\prime}\right)}{8}, \\
& J_{2}\left(B_{I}\right) \\
& =\frac{\left(a_{1 \mu}+a_{2 \mu}\right)-\left(a_{3 \mu}+a_{4 \mu}\right)-\left(a_{1 \nu}^{\prime}+a_{2 \nu}^{\prime}\right)+\left(a_{3 v}^{\prime}+a_{4 \nu}^{\prime}\right)}{8} \\
& \quad+\epsilon .
\end{aligned}
$$

Therefore $J_{2}\left(B_{I}\right)>J_{2}\left(A_{I}\right)$; hence $B_{I}>A_{I}$.

Example 28. Let $A_{I}=\left\langle\left(a_{1 \mu}, a_{2 \mu}, a_{3 \mu}, a_{4 \mu}\right),\left(a_{1 \nu}^{\prime}, a_{2 \nu}^{\prime}, a_{3 \nu}^{\prime}, a_{4 \nu}^{\prime}\right)\right\rangle$ and $B_{I}=\left\langle\left(a_{1 \mu}+\epsilon, a_{2 \mu}+\epsilon, a_{3 \mu}+\epsilon, a_{4 \mu}+\epsilon\right),\left(a_{1 \nu}^{\prime}+\epsilon / 2, a_{2 \nu}^{\prime}+\right.\right.$ $\left.\left.\epsilon / 2, a_{3 \nu}^{\prime}+\epsilon / 2, a_{4 v}^{\prime}+\epsilon / 2\right)\right\rangle \in \operatorname{TrIFN}$ and $\epsilon \in[0,1]$ but not in $C_{2}$. Now

$$
\begin{aligned}
& J_{1}\left(A_{I}\right)=J_{1}\left(B_{I}\right) \\
& =\frac{\left(a_{1 \mu}+a_{2 \mu}\right)-\left(a_{3 \mu}+a_{4 \mu}\right)+\left(a_{1 \nu}^{\prime}+a_{2 \nu}^{\prime}\right)-\left(a_{3 \nu}^{\prime}+a_{4 \nu}^{\prime}\right)}{8}, \\
& J_{2}\left(A_{I}\right)=J_{2}\left(B_{I}\right) \\
& =\frac{\left(a_{1 \mu}+a_{2 \mu}\right)-\left(a_{3 \mu}+a_{4 \mu}\right)-\left(a_{1 \nu}^{\prime}+a_{2 \nu}^{\prime}\right)+\left(a_{3 \nu}^{\prime}+a_{4 \nu}^{\prime}\right)}{8} .
\end{aligned}
$$

But $A_{I} \neq B_{I}$.

Pictorial representation of this example is given in Figure 4 .

From Example 28 it is easy to see that $J_{1}$ and $J_{2}$ are not enough to cover the entire class of TrIFNs. Therefore in the next subsection we are introducing a new score function which covers some more subclasses of TrIFNs that cannot be covered by $J_{1}$ and $J_{2}$.

3.3. Incomplete Score of a Trapezoidal Intuitionistic Fuzzy Number. In this subsection, another class of TrIFNs is introduced. The incomplete score function on this class of 
trapezoidal intuitionistic fuzzy numbers is defined and some of its properties are studied using illustrative examples.

Definition 29. Let $A_{I}$ and $B_{I}$ be two TrIFNs.

A special subclass $C_{3}$ of the set of TrIFNs consist of TrIFNs for which every pair of $A_{I}$ and $B_{I}$ are with $a_{1 \mu} \leq b_{1 \mu}, a_{2 \mu} \leq$ $b_{2 \mu}, a_{3 \mu} \leq b_{3 \mu}, a_{4 \mu} \leq b_{4 \mu}$ and $a_{1 \nu}^{\prime} \geq b_{1 \nu}^{\prime}, a_{2 \nu}^{\prime} \geq b_{2 \nu}^{\prime}, a_{3 \nu}^{\prime} \geq$ $b_{3 v}^{\prime}, a_{4 v}^{\prime} \geq b_{4 v}^{\prime}$. The incomplete relation on $C_{3}$ is denoted as $\subseteq$ and defined as follows: if $A_{I}, B_{I} \in C_{3} \subset$ TrIFN such that $A_{I} \subseteq B_{I}$ then $a_{1 \mu} \leq b_{1 \mu}, a_{2 \mu} \leq b_{2 \mu}, a_{3 \mu} \leq b_{3 \mu}, a_{4 \mu} \leq b_{4 \mu}$ and $a_{1 v}^{\prime} \geq b_{1 v}^{\prime}, a_{2 v}^{\prime} \geq b_{2 v}^{\prime}, a_{3 v}^{\prime} \geq b_{3 \nu}^{\prime}, a_{4 v}^{\prime} \geq b_{4 v}^{\prime}$.

If $A_{I} \subset B_{I}$ then one of the above inequalities becomes strict inequality.

Note 5. By Definition 29, we note that any pair of members of $\mathrm{C}_{3}$ are related under $C$.

The incomplete score function which measures the completeness is defined as follows.

Definition 30. Let $A_{I}$ be a trapezoidal intuitionistic fuzzy number. Then the incomplete score of $A_{I}$ is defined by

$$
\begin{aligned}
J_{3}\left(A_{I}\right) & \\
= & \int_{0}^{1} \frac{-\left[{ }^{\alpha} \mu_{A_{I_{L}}}+{ }^{\alpha} \mu_{A_{I_{U}}}\right]}{4} d \alpha \\
& +\int_{0}^{1} \frac{\left[{ }^{\beta} v_{A_{I_{L}}}+{ }^{\beta} v_{A_{I_{U}}}\right]}{4} d \beta \\
= & \frac{\left(a_{1 v}^{\prime}+a_{2 v}^{\prime}+a_{3 v}^{\prime}+a_{4 \nu}^{\prime}\right)-\left(a_{1 \mu}+a_{2 \mu}+a_{3 \mu}+a_{4 \mu}\right)}{8} .
\end{aligned}
$$

The proofs of the following propositions are immediate from the above definition and hence they are omitted.

Proposition 31. For any real number $r \in[0,1], J_{3}(r)=0$.

Proposition 32. If $A_{I}=\left(a_{1 \mu}, a_{2 \mu}, a_{3 \mu}, a_{4 \mu}\right)$ is a trapezoidal fuzzy number, then $J_{3}\left(A_{I}\right)=0$.

Proposition 33. Let $A_{I}=\left\langle\left(a_{1 \mu}, a_{2 \mu}, a_{3 \mu}\right),\left(a_{1 \nu}^{\prime}, a_{2 v}^{\prime}, a_{3 \nu}^{\prime}\right)\right\rangle$ be a triangular intuitionistic fuzzy number. Then

$$
J_{3}\left(A_{I}\right)=\frac{\left(a_{1 \nu}^{\prime}+2 a_{2 \nu}^{\prime}+a_{3 \nu}^{\prime}\right)-\left(a_{1 \mu}+2 a_{2 \mu}+a_{3 \mu}\right)}{8} .
$$

Proposition 34. Let $A_{I}=\left(\left[a_{1 \mu}, a_{2 \mu}\right],\left[a_{1 v}^{\prime}, a_{2 v}^{\prime}\right]\right)$ be an interval valued intuitionistic fuzzy number. Then

$$
J_{3}\left(A_{I}\right)=\frac{\left(a_{1 \nu}^{\prime}+a_{2 \nu}^{\prime}\right)-\left(a_{1 \mu}+a_{2 \mu}\right)}{4} .
$$

Theorem 35. Let $A_{I}, B_{I} \in C_{3}$. If $A_{I} \subset B_{I}$ then $J_{3}\left(A_{I}\right)>$ $J_{3}\left(B_{I}\right)$.

Proof. Let us assume that $A_{I} \subset B_{I}$. We claim that $J_{3}\left(A_{I}\right)-$ $J_{3}\left(B_{I}\right)>0$.
By Definition 29, we have

$$
\begin{aligned}
& a_{1 \mu} \leq b_{1 \mu}, \\
& a_{2 \mu} \leq b_{2 \mu}, \\
& a_{3 \mu} \leq b_{3 \mu}, \\
& a_{4 \mu} \leq b_{4 \mu}, \\
& a_{1 v}^{\prime} \geq b_{1 \nu}^{\prime}, \\
& a_{2 v}^{\prime} \geq b_{2 \gamma}^{\prime}, \\
& a_{3 v}^{\prime} \geq b_{3 \nu}^{\prime}, \\
& a_{4 v}^{\prime} \geq b_{4 v}^{\prime} .
\end{aligned}
$$

Now,

$$
\begin{gathered}
8\left(J_{3}\left(A_{I}\right)-J_{3}\left(B_{I}\right)\right)=\left[\left(b_{1 \mu}-a_{1 \mu}\right)+\left(b_{2 \mu}-a_{2 \mu}\right)\right. \\
\left.+\left(b_{3 \mu}-a_{3 \mu}\right)+\left(b_{4 \mu}-a_{4 \mu}\right)\right]+\left[\left(a_{1 \nu}^{\prime}-b_{1 \nu}^{\prime}\right)\right. \\
\left.+\left(a_{2 \nu}^{\prime}-b_{2 \nu}^{\prime}\right)+\left(a_{3 \nu}^{\prime}-b_{3 \nu}^{\prime}\right)+\left(a_{4 \nu}^{\prime}-b_{4 \nu}^{\prime}\right)\right] .
\end{gathered}
$$

From (19) it is very easy to see that all the terms in (20) are positive. Therefore their sum is also positive. From Definition 29, we know that at least one of the above inequalities in (19) becomes strict inequality and hence we get $J_{3}\left(A_{I}\right)-$ $J_{3}\left(B_{I}\right)>0$, hence the proof.

Theorem 36. Let $A_{I}, B_{I} \in C_{3}$. If $J_{3}\left(A_{I}\right)=J_{3}\left(B_{I}\right)$ then $A_{I}=$ $B_{I}$.

Proof. Let us assume $J_{3}\left(A_{I}\right)=J_{2}\left(B_{I}\right)$. We prove that $A_{I}=B_{I}$. By Definition 29, without loss of generality, we have

$$
\begin{aligned}
& a_{1 \mu} \leq b_{1 \mu}, \\
& a_{2 \mu} \leq b_{2 \mu}, \\
& a_{3 \mu} \leq b_{3 \mu}, \\
& a_{4 \mu} \leq b_{4 \mu}, \\
& a_{1 \nu}^{\prime} \geq b_{1 v}^{\prime}, \\
& a_{2 v}^{\prime} \geq b_{2 v}^{\prime}, \\
& a_{3 v}^{\prime} \geq b_{3 v}^{\prime}, \\
& a_{4 v}^{\prime} \geq b_{4 v}^{\prime} .
\end{aligned}
$$

Now,

$$
\begin{gathered}
8\left(J_{3}\left(A_{I}\right)-J_{3}\left(B_{I}\right)\right)=\left[\left(b_{1 \mu}-a_{1 \mu}\right)+\left(b_{2 \mu}-a_{2 \mu}\right)\right. \\
\left.+\left(b_{3 \mu}-a_{3 \mu}\right)+\left(b_{4 \mu}-a_{4 \mu}\right)\right]+\left[\left(a_{1 \nu}^{\prime}-b_{1 \nu}^{\prime}\right)\right. \\
\left.\quad+\left(a_{2 \nu}^{\prime}-b_{2 \nu}^{\prime}\right)+\left(a_{3 \nu}^{\prime}-b_{3 \nu}^{\prime}\right)+\left(a_{4 \nu}^{\prime}-b_{4 \nu}^{\prime}\right)\right]=0 .
\end{gathered}
$$


Therefore from (21) and (22), it is clear that all the terms in (22) are positive and therefore their sum gives zero only when each term is equal to zero. Hence $a_{1 \mu}=b_{1 \mu}, a_{2 \mu}=b_{2 \mu}, a_{3 \mu}=$ $b_{3 \mu}, a_{4 \mu}=b_{4 \mu}$ and $a_{1 v}^{\prime}=b_{1 v}^{\prime}, a_{2 v}^{\prime}=b_{2 v}^{\prime}, a_{3 v}^{\prime}=b_{3 v}^{\prime}, a_{4 v}^{\prime}=b_{4 v}^{\prime}$. Hence $A_{I}=B_{I}$.

Note 6. The incomplete score can be calculated to any TrIFN. But $J_{3}$ gives total order on $C_{3}$.

Definition 37. If $J_{3}\left(A_{I}\right)>J_{3}\left(B_{I}\right)\left(J_{3}\left(A_{I}\right)<J_{3}\left(B_{I}\right)\right)$, then $A_{I}>$ $B_{I}\left(A_{I}<B_{I}\right)$.

The following example is used to explain the ranking procedure defined in Definition 37.

Example 38. Let $A_{I}=\langle(0.15,0.3,0.4,0.45),(0.1,0.25,0.45$, $0.6)\rangle$ and $B_{I}=\langle(0.2,0.4,0.45,0.55),(0,0.15,0.45,0.55)\rangle \epsilon$ $C_{3}$. Now $J_{3}\left(A_{I}\right)=0.0125$ and $J_{3}\left(B_{I}\right)=-0.05625, J_{3}\left(A_{I}\right)>$ $J_{3}\left(B_{I}\right)$.

Hence $A_{I}>B_{I}$.

The inefficiency of the score functions $J_{1}$ and $J_{2}$ in discriminating any two arbitrary TrIFNs and the ability of $J_{3}$ in comparing arbitrary TrIFNs is shown in the following example.

Example 39. Let $A_{I}=\left\langle\left(a_{1 \mu}, a_{2 \mu}, a_{3 \mu}, a_{4 \mu}\right),\left(a_{1 \nu}^{\prime}, a_{2 v}^{\prime}, a_{3 v}^{\prime}, a_{4 \nu}^{\prime}\right)\right\rangle$ and $B_{I}=\left\langle\left(a_{1 \mu}+\epsilon, a_{2 \mu}+\epsilon, a_{3 \mu}+\epsilon, a_{4 \mu}+\epsilon\right),\left(a_{1 v}^{\prime}+\epsilon / 2, a_{2 v}^{\prime}+\right.\right.$ $\left.\left.\epsilon / 2, a_{3 \nu}^{\prime}+\epsilon / 2, a_{4 v}^{\prime}+\epsilon / 2\right)\right\rangle \in \operatorname{TrIFN}$ and $\epsilon \in[0,1]$.

Now

$$
\begin{aligned}
& J_{1}\left(A_{I}\right)=J_{1}\left(B_{I}\right) \\
& =\frac{\left(a_{1 \mu}+a_{2 \mu}\right)-\left(a_{3 \mu}+a_{4 \mu}\right)+\left(a_{1 \nu}^{\prime}+a_{2 \nu}^{\prime}\right)-\left(a_{3 \nu}^{\prime}+a_{4 \nu}^{\prime}\right)}{8}, \\
& J_{2}\left(A_{I}\right)=J_{2}\left(B_{I}\right) \\
& =\frac{\left(a_{1 \mu}+a_{2 \mu}\right)-\left(a_{3 \mu}+a_{4 \mu}\right)-\left(a_{1 \nu}^{\prime}+a_{2 \nu}^{\prime}\right)+\left(a_{3 \nu}^{\prime}+a_{4 \nu}^{\prime}\right)}{8} .
\end{aligned}
$$

But

$$
\begin{aligned}
& J_{3}\left(A_{I}\right) \\
& =\frac{\left(a_{1 v}^{\prime}+a_{2 v}^{\prime}+a_{3 v}^{\prime}+a_{4 v}^{\prime}\right)-\left(a_{1 \mu}+a_{2 \mu}+a_{3 \mu}+a_{4 \mu}\right)}{8}, \\
& J_{3}\left(B_{I}\right) \\
& =\frac{\left(a_{1 v}^{\prime}+a_{2 v}^{\prime}+a_{3 v}^{\prime}+a_{4 v}^{\prime}\right)-\left(a_{1 \mu}+a_{2 \mu}+a_{3 \mu}+a_{4 \mu}\right)}{8} \\
& \quad+\frac{\epsilon}{4} \Longrightarrow \\
& J_{3}\left(B_{I}\right)>J_{3}\left(A_{I}\right) .
\end{aligned}
$$

Hence $B_{I}>A_{I}$.

The inefficiency of the score functions $J_{1}$ to $J_{3}$ in the task of comparing TrIFNs is explained in the following example.

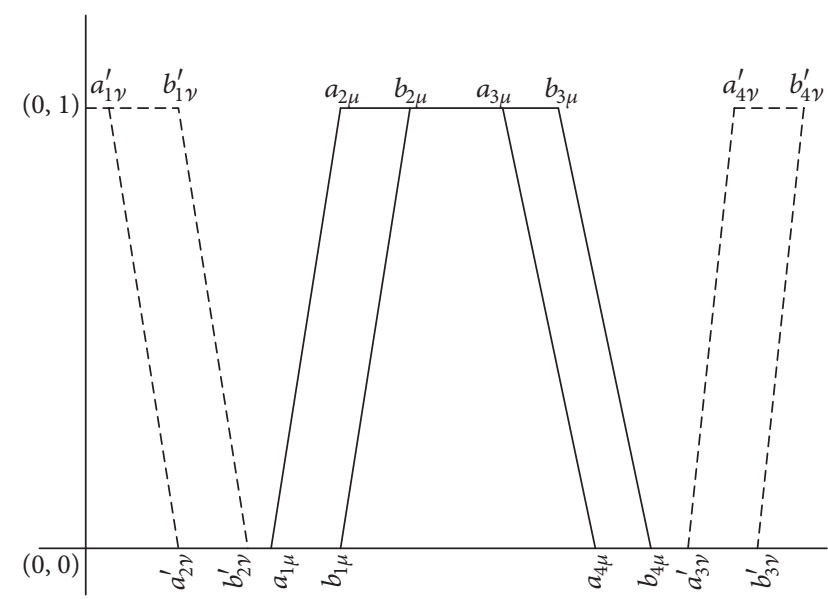

FIgURE 5: Pictorial representation of Example 40.

Example 40. Let $A_{I}=\left\langle\left(a_{1 \mu}, a_{2 \mu}, a_{3 \mu}, a_{4 \mu}\right),\left(a_{1 v}^{\prime}, a_{2 v}^{\prime}, a_{3 v}^{\prime}, a_{4 v}^{\prime}\right)\right\rangle$ and $B_{I}=\left\langle\left(a_{1 \mu}+\epsilon, a_{2 \mu}+\epsilon, a_{3 \mu}+\epsilon, a_{4 \mu}+\epsilon\right),\left(a_{1 \nu}^{\prime}+\epsilon, a_{2 \nu}^{\prime}+\epsilon, a_{3 \nu}^{\prime}+\right.\right.$ $\left.\left.\epsilon, a_{4 \nu}^{\prime}+\epsilon\right)\right\rangle \in \operatorname{TrIFN}$ and $\epsilon \in[0,1]$ but not in $C_{3}$.

Now

$$
\begin{aligned}
& J_{1}\left(A_{I}\right)=J_{1}\left(B_{I}\right) \\
& =\frac{\left(a_{1 \mu}+a_{2 \mu}\right)-\left(a_{3 \mu}+a_{4 \mu}\right)+\left(a_{1 \nu}^{\prime}+a_{2 \nu}^{\prime}\right)-\left(a_{3 \nu}^{\prime}+a_{4 \nu}^{\prime}\right)}{8}, \\
& J_{2}\left(A_{I}\right)=J_{2}\left(B_{I}\right) \\
& =\frac{\left(a_{1 \mu}+a_{2 \mu}\right)-\left(a_{3 \mu}+a_{4 \mu}\right)-\left(a_{1 \nu}^{\prime}+a_{2 \nu}^{\prime}\right)+\left(a_{3 \nu}^{\prime}+a_{4 \nu}^{\prime}\right)}{8}, \\
& J_{3}\left(A_{I}\right)=J_{3}\left(B_{I}\right) \\
& =\frac{\left(a_{1 v}^{\prime}+a_{2 v}^{\prime}+a_{3 \nu}^{\prime}+a_{4 v}^{\prime}\right)-\left(a_{1 \mu}+a_{2 \mu}+a_{3 \mu}+a_{4 \mu}\right)}{8} .
\end{aligned}
$$

But $A_{I} \neq B_{I}$.

Pictorial representation of this example is given in Figure 5.

Example 40 shows that $J_{1}, J_{2}$, and $J_{3}$ cannot be sufficient to cover the entire class of TrIFNs and the class of TrIFNs in the above example excite us to define new score function that can fill the subclass of TrIFNs which cannot be covered by $J_{1}$, $J_{2}$, and $J_{3}$.

3.4. Accuracy Score of a Trapezoidal Intuitionistic Fuzzy Number. In this subsection, a new subclass of TrIFNs using accuracy relation is introduced and the accuracy score function on this class of trapezoidal intuitionistic fuzzy numbers is defined and some of its properties are studied using illustrative examples. 
Definition 41. Let $A_{I}$ and $B_{I}$ be two TrIFNs.

A special subclass $C_{4}$ of the set of TrIFNs consist of TrIFNs for which every pair of $A_{I}$ and $B_{I}$ are with $a_{1 \mu} \leq b_{1 \mu}, a_{2 \mu} \leq$ $b_{2 \mu}, a_{3 \mu} \leq b_{3 \mu}, a_{4 \mu} \leq b_{4 \mu}$ and $a_{1 v}^{\prime} \leq b_{1 v}^{\prime}, a_{2 v}^{\prime} \leq b_{2 v}^{\prime}, a_{3 \nu}^{\prime} \leq$ $b_{3 v}^{\prime}, a_{4 v}^{\prime} \leq b_{4 v}^{\prime}$. The accuracy relation on $C_{4}$ is denoted as $\preceq$ and defined as follows: if $A_{I}, B_{I} \in C_{4} \subset$ TrIFN such that $A_{I} \preceq B_{I}$ then $a_{1 \mu} \leq b_{1 \mu}, a_{2 \mu} \leq b_{2 \mu}, a_{3 \mu} \leq b_{3 \mu}, a_{4 \mu} \leq b_{4 \mu}$ and $a_{1 v}^{\prime} \leq b_{1 v}^{\prime}, a_{2 v}^{\prime} \leq b_{2 v}^{\prime}, a_{3 v}^{\prime} \leq b_{3 v}^{\prime}, a_{4 v}^{\prime} \leq b_{4 v}^{\prime}$.

If $A_{I} \prec B_{I}$ then one of the above inequalities becomes strict inequality.

Note 7. By Definition 41, we note that any pair of members of $\mathrm{C}_{4}$ are related under $\prec$.

The score function which measures the accuracy is defined as follows.

Definition 42. Let $A_{I}$ be a trapezoidal intuitionistic fuzzy number. Then the accuracy score of $A_{I}$ is defined by

$$
\begin{aligned}
J_{4} & \left(A_{I}\right) \\
= & \int_{0}^{1} \frac{\left[{ }^{\alpha} \mu_{A_{I_{L}}}+{ }^{\alpha} \mu_{A_{I_{U}}}\right]}{4} d \alpha+\int_{0}^{1} \frac{\left[{ }^{\beta} v_{A_{I_{L}}}+{ }^{\beta} v_{A_{I_{U}}}\right]}{4} d \beta \\
= & \frac{\left(a_{1 \mu}+a_{2 \mu}+a_{3 \mu}+a_{4 \mu}\right)+\left(a_{1 v}^{\prime}+a_{2 v}^{\prime}+a_{3 v}^{\prime}+a_{4 v}^{\prime}\right)}{8} .
\end{aligned}
$$

The proofs of the following propositions are immediate from Definition 42 and hence they are omitted.

Proposition 43. For any real number $r \in[0,1], J_{4}(r)=r$.

Proposition 44. For any trapezoidal fuzzy number $A_{I}=$ $\left(a_{1 \mu}, a_{2 \mu}, a_{3 \mu}, a_{4 \mu}\right), J_{4}\left(A_{I}\right)=\left(a_{1 \mu}+a_{2 \mu}+a_{3 \mu}+a_{4 \mu}\right) / 4$.

Proposition 45. Let $A_{I}=\left\langle\left(a_{1 \mu}, a_{2 \mu}, a_{3 \mu}\right),\left(a_{1 v}^{\prime}, a_{2 v}^{\prime}, a_{3 v}^{\prime}\right)\right\rangle$ be a triangular intuitionistic fuzzy number. Then $J_{4}\left(A_{I}\right)=\left(\left(a_{1 \mu}+\right.\right.$ $\left.\left.2 a_{2 \mu}+a_{3 \mu}\right)+\left(a_{1 v}^{\prime}+2 a_{2 v}^{\prime}+a_{3 \nu}^{\prime}\right)\right) / 8$.

Proposition 46. Let $A_{I}=\left(\left[a_{1 \mu}, a_{2 \mu}\right],\left[a_{1 v}^{\prime}, a_{2 \nu}^{\prime}\right]\right)$ be an interval valued intuitionistic fuzzy number. Then $J_{4}\left(A_{I}\right)=\left(\left(a_{1 \mu}+a_{2 \mu}\right)+\right.$ $\left.\left(a_{1 v}^{\prime}+a_{2 v}^{\prime}\right)\right) / 4$.

Note 8. The accuracy score can be calculated to any TrIFN. But $J_{4}$ gives total order on $C_{4}$ which is proved in Theorems 47 and 48 .

Theorem 47. Let $A_{I}, B_{I} \in C_{4}$. If $A_{I} \prec B_{I}$ then $J_{4}\left(A_{I}\right)<$ $J_{4}\left(B_{I}\right)$.

Proof. Let us assume that $A_{I} \prec B_{I}$. We claim that $J_{4}\left(B_{I}\right)-$ $J_{4}\left(A_{I}\right)>0$.

By Definition 41, we have

$$
\begin{aligned}
& a_{1 \mu} \leq b_{1 \mu}, \\
& a_{2 \mu} \leq b_{2 \mu}, \\
& a_{3 \mu} \leq b_{3 \mu},
\end{aligned}
$$

$$
\begin{aligned}
& a_{4 \mu} \leq b_{4 \mu}, \\
& a_{1 v}^{\prime} \leq b_{1 v}^{\prime}, \\
& a_{2 v}^{\prime} \leq b_{2 v}^{\prime}, \\
& a_{3 v}^{\prime} \leq b_{3 v}^{\prime}, \\
& a_{4 v}^{\prime} \leq b_{4 v}^{\prime} .
\end{aligned}
$$

Now,

$$
\begin{gathered}
8\left(J_{4}\left(B_{I}\right)-J_{4}\left(A_{I}\right)\right)=\left[\left(b_{1 \mu}-a_{1 \mu}\right)+\left(b_{2 \mu}-a_{2 \mu}\right)\right. \\
\left.\quad+\left(b_{3 \mu}-a_{3 \mu}\right)+\left(b_{4 \mu}-a_{4 \mu}\right)\right]+\left[\left(b_{1 v}^{\prime}-a_{1 v}^{\prime}\right)\right. \\
\left.\quad+\left(b_{2 v}^{\prime}-a_{2 v}^{\prime}\right)+\left(b_{3 \nu}^{\prime}-a_{3 v}^{\prime}\right)+\left(b_{4 v}^{\prime}-a_{4 v}^{\prime}\right)\right] .
\end{gathered}
$$

From (27) it is very easy to see that all the terms in (28) are positive. Therefore their sum is also positive. From Definition 41, we know that at least one of the above inequalities in (27) becomes strict inequality and hence we get $J_{4}\left(B_{I}\right)-$ $J_{4}\left(A_{I}\right)>0$.

Theorem 48. Let $A_{I}, B_{I} \in C_{4}$ such that $J_{4}\left(A_{I}\right)=J_{4}\left(B_{I}\right)$; then $A_{I}=B_{I}$.

Proof. Let us assume $J_{4}\left(A_{I}\right)=J_{4}\left(B_{I}\right)$. We claim that $A_{I}=B_{I}$. By Definition 41, without loss of generality, we have

$$
\begin{aligned}
& a_{1 \mu} \leq b_{1 \mu}, \\
& a_{2 \mu} \leq b_{2 \mu}, \\
& a_{3 \mu} \leq b_{3 \mu}, \\
& a_{4 \mu} \leq b_{4 \mu}, \\
& a_{1 v}^{\prime} \geq b_{1 v}^{\prime}, \\
& a_{2 v}^{\prime} \geq b_{2 v}^{\prime}, \\
& a_{3 v}^{\prime} \geq b_{3 v}^{\prime}, \\
& a_{4 v}^{\prime} \geq b_{4 v}^{\prime} .
\end{aligned}
$$

Now,

$$
\begin{gathered}
8\left(J_{4}\left(B_{I}\right)-J_{4}\left(A_{I}\right)\right)=\left[\left(b_{1 \mu}-a_{1 \mu}\right)+\left(b_{2 \mu}-a_{2 \mu}\right)\right. \\
\left.\quad+\left(b_{3 \mu}-a_{3 \mu}\right)+\left(b_{4 \mu}-a_{4 \mu}\right)\right]+\left[\left(b_{1 v}^{\prime}-a_{1 v}^{\prime}\right)\right. \\
\left.\quad+\left(b_{2 v}^{\prime}-a_{2 v}^{\prime}\right)+\left(b_{3 v}^{\prime}-a_{3 \nu}^{\prime}\right)+\left(b_{4 \nu}^{\prime}-a_{4 v}^{\prime}\right)\right]=0 .
\end{gathered}
$$

Therefore from (29) and (30), it is clear that all the terms in (30) are positive and therefore their sum gives zero only when each term is equal to zero. Hence $a_{1 \mu}=b_{1 \mu}, a_{2 \mu}=b_{2 \mu}, a_{3 \mu}=$ $b_{3 \mu}, a_{4 \mu}=b_{4 \mu}$ and $a_{1 v}^{\prime}=b_{1 v}^{\prime}, a_{2 v}^{\prime}=b_{2 v}^{\prime}, a_{3 v}^{\prime}=b_{3 v}^{\prime}, a_{4 v}^{\prime}=b_{4 v}^{\prime}$. Hence $A_{I}=B_{I}$. 
Definition 49. If $J_{4}\left(A_{I}\right)>J_{4}\left(B_{I}\right)\left(J_{4}\left(A_{I}\right)<J_{4}\left(B_{I}\right)\right)$ then $A_{I}>$ $B_{I}\left(A_{I}<B_{I}\right)$.

Ranking procedure defined in Definition 49 is illustrated in the following example.

Example 50. Let $A_{I}=\langle(0.1,0.25,0.37,0.49),(0.1,0.2,0.4$, $0.6)\rangle$ and $B_{I}=\langle(0.2,0.37,0.47,0.6),(0.15,0.32,0.52,0.7)\rangle \epsilon$ $C_{4}$. Now $J_{4}\left(A_{I}\right)=0.31375$ and $J_{4}\left(B_{I}\right)=0.41625, J_{4}\left(A_{I}\right)<$ $J_{4}\left(B_{I}\right)$.

Hence $A_{I}<B_{I}$.

The relative importance of the score function $J_{4}$ in discriminating two different TrIFNs when $J_{1}, J_{2}$, and $J_{3}$ fail to discriminate them is explained by Example 51 .

Example 51. Let $A_{I}=\left\langle\left(a_{1 \mu}, a_{2 \mu}, a_{3 \mu}, a_{4 \mu}\right),\left(a_{1 v}^{\prime}, a_{2 v}^{\prime}, a_{3 v}^{\prime}, a_{4 v}^{\prime}\right)\right\rangle$ and $B_{I}=\left\langle\left(a_{1 \mu}+\epsilon, a_{2 \mu}+\epsilon, a_{3 \mu}+\epsilon, a_{4 \mu}+\epsilon\right),\left(a_{1 v}^{\prime}+\epsilon, a_{2 v}^{\prime}+\right.\right.$ $\left.\left.\epsilon, a_{3 \nu}^{\prime}+\epsilon, a_{4 \nu}^{\prime}+\epsilon\right)\right\rangle \in \operatorname{TrIFN}$, and $\epsilon \in[0,1]$.

Now

$$
\begin{aligned}
& J_{1}\left(A_{I}\right)=J_{1}\left(B_{I}\right) \\
& =\frac{\left(a_{1 \mu}+a_{2 \mu}\right)-\left(a_{3 \mu}+a_{4 \mu}\right)+\left(a_{1 v}^{\prime}+a_{2 v}^{\prime}\right)-\left(a_{3 \nu}^{\prime}+a_{4 v}^{\prime}\right)}{8}, \\
& J_{2}\left(A_{I}\right)=J_{2}\left(B_{I}\right) \\
& =\frac{\left(a_{1 \mu}+a_{2 \mu}\right)-\left(a_{3 \mu}+a_{4 \mu}\right)-\left(a_{1 \nu}^{\prime}+a_{2 v}^{\prime}\right)+\left(a_{3 \nu}^{\prime}+a_{4 v}^{\prime}\right)}{8}, \\
& J_{3}\left(A_{I}\right)=J_{3}\left(B_{I}\right) \\
& =\frac{\left(a_{1 \nu}^{\prime}+a_{2 v}^{\prime}+a_{3 \nu}^{\prime}+a_{4 \nu}^{\prime}\right)-\left(a_{1 \mu}+a_{2 \mu}+a_{3 \mu}+a_{4 \mu}\right)}{8} .
\end{aligned}
$$

But

$$
\begin{aligned}
& J_{4}\left(A_{I}\right) \\
& =\frac{\left(a_{1 \mu}+a_{2 \mu}+a_{3 \mu}+a_{4 \mu}\right)+\left(a_{1 v}^{\prime}+a_{2 v}^{\prime}+a_{3 \nu}^{\prime}+a_{4 v}^{\prime}\right)}{8}, \\
& J_{4}\left(B_{I}\right) \\
& =\frac{\left(a_{1 \mu}+a_{2 \mu}+a_{3 \mu}+a_{4 \mu}\right)+\left(a_{1 v}^{\prime}+a_{2 v}^{\prime}+a_{3 \nu}^{\prime}+a_{4 v}^{\prime}\right)}{8} \\
& \quad+\epsilon \Longrightarrow \\
& J_{4}\left(A_{I}\right)<J_{4}\left(B_{I}\right) .
\end{aligned}
$$

Hence $A_{I}<B_{I}$.

Example 52. Let $A_{I}=\left\langle\left(a_{1 \mu}, a_{2 \mu}, a_{3 \mu}, a_{4 \mu}\right),\left(a_{1 v}^{\prime}, a_{2 v}^{\prime}, a_{3 v}^{\prime}, a_{4 v}^{\prime}\right)\right\rangle$ and $B_{I}=\left\langle\left(a_{1 \mu}+\epsilon, a_{2 \mu}-\epsilon, a_{3 \mu}+\epsilon, a_{4 \mu}-\epsilon\right),\left(a_{1 v}^{\prime}+\epsilon, a_{2 v}^{\prime}-\right.\right.$ $\left.\left.\epsilon, a_{3 v}^{\prime}+\epsilon, a_{4 v}^{\prime}-\epsilon\right)\right\rangle \in \operatorname{TrIFN}$ and $\epsilon \in[0,1]$ but not in $C_{4}$.

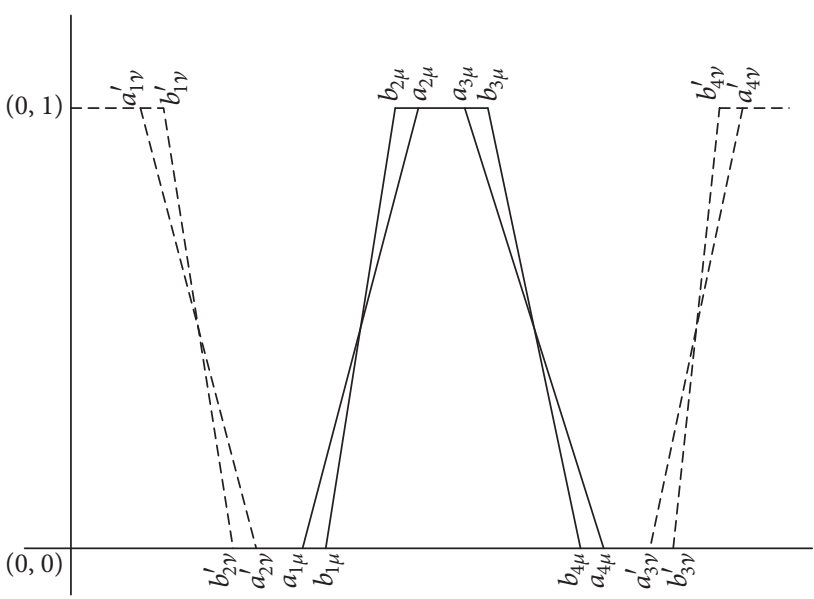

FIgURE 6: Pictorial representation of Example 52.

Now

$$
\begin{aligned}
& J_{1}\left(A_{I}\right)=J_{1}\left(B_{I}\right) \\
& =\frac{\left(a_{1 \mu}+a_{2 \mu}\right)-\left(a_{3 \mu}+a_{4 \mu}\right)+\left(a_{1 v}^{\prime}+a_{2 v}^{\prime}\right)-\left(a_{3 v}^{\prime}+a_{4 v}^{\prime}\right)}{8}, \\
& J_{2}\left(A_{I}\right)=J_{2}\left(B_{I}\right) \\
& =\frac{\left(a_{1 \mu}+a_{2 \mu}\right)-\left(a_{3 \mu}+a_{4 \mu}\right)-\left(a_{1 v}^{\prime}+a_{2 v}^{\prime}\right)+\left(a_{3 \nu}^{\prime}+a_{4 v}^{\prime}\right)}{8}, \\
& J_{3}\left(A_{I}\right)=J_{3}\left(B_{I}\right) \\
& =\frac{\left(a_{1 v}^{\prime}+a_{2 \nu}^{\prime}+a_{3 \nu}^{\prime}+a_{4 \nu}^{\prime}\right)-\left(a_{1 \mu}+a_{2 \mu}+a_{3 \mu}+a_{4 \mu}\right)}{8}, \\
& J_{4}\left(A_{I}\right)=J_{4}\left(B_{I}\right) \\
& =\frac{\left(a_{1 \mu}+a_{2 \mu}+a_{3 \mu}+a_{4 \mu}\right)+\left(a_{1 v}^{\prime}+a_{2 v}^{\prime}+a_{3 \nu}^{\prime}+a_{4 v}^{\prime}\right)}{8} .
\end{aligned}
$$

But $A_{I} \neq B_{I}$.

Pictorial representation of this example is given in Figure 6.

Example 52 shows that even $J_{1}, J_{2}, J_{3}$, and $J_{4}$ altogether are not enough to cover the entire class of TrIFNs; the class of TrIFNs in the above example excite us to define new score function that can cover the class of TrIFNs which cannot be covered by $J_{1}, J_{2}, J_{3}$, and $J_{4}$.

3.5. Spread Score of a Trapezoidal Intuitionistic Fuzzy Number. In this subsection, another subclass of TrIFNs is introduced. The spread score function on this class of trapezoidal intuitionistic fuzzy numbers is defined and some of its properties are studied using illustrative examples.

Definition 53. Let $A_{I}$ and $B_{I}$ be two TrIFNs.

A special subclass $C_{5}$ of the set of TrIFNs consist of TrIFNs for which every pair of $A_{I}$ and $B_{I}$ are with $a_{1 \mu} \geq b_{1 \mu}, a_{2 \mu} \leq$ $b_{2 \mu}, a_{3 \mu} \geq b_{3 \mu}, a_{4 \mu} \leq b_{4 \mu}$ and $a_{1 v}^{\prime} \geq b_{1 v}^{\prime}, a_{2 v}^{\prime} \leq b_{2 v}^{\prime}, a_{3 v}^{\prime} \geq$ $b_{3 v}^{\prime}, a_{4 v}^{\prime} \leq b_{4 v}^{\prime}$. The preference relation on $C_{5}$ is denoted as 
$\unlhd$ and defined as follows: if $A_{I}, B_{I} \in C_{5} \subset$ TrIFN such that $A_{I} \unlhd B_{I}$ then $a_{1 \mu} \geq b_{1 \mu}, a_{2 \mu} \leq b_{2 \mu}, a_{3 \mu} \geq b_{3 \mu}, a_{4 \mu} \leq b_{4 \mu}$ and $a_{1 v}^{\prime} \geq b_{1 v}^{\prime}, a_{2 v}^{\prime} \leq b_{2 v}^{\prime}, a_{3 v}^{\prime} \geq b_{3 v}^{\prime}, a_{4 v}^{\prime} \leq b_{4 v}^{\prime}$. If $A_{I} \triangleleft B_{I}$ then one of the above inequalities becomes strict inequality.

Note 9. By Definition 53, we note that any pair of members of $C_{5}$ are related under $\triangleleft$.

The score function which measures the spread is defined as follows.

Definition 54. Let $A_{I}$ be a trapezoidal intuitionistic fuzzy number. Then the spread score of $A_{I}$ is defined by

$$
\begin{aligned}
& J_{5}\left(A_{I}\right) \\
& =\frac{\left(a_{1 \mu}+a_{3 \mu}\right)-\left(a_{2 \mu}+a_{4 \mu}\right)-\left(a_{2 \nu}^{\prime}+a_{4 \nu}^{\prime}\right)+\left(a_{1 v}^{\prime}+a_{3 \nu}^{\prime}\right)}{8} .
\end{aligned}
$$

The proofs of the following propositions are immediate from Definition 54 and hence they are omitted.

Proposition 55. For any real number $r \in[0,1], J_{5}(r)=0$.

Proposition 56. If $A_{I}=\left(a_{1 \mu}, a_{2 \mu}, a_{3 \mu}, a_{4 \mu}\right)$ is a trapezoidal fuzzy number, then $J_{5}\left(A_{I}\right)=\left(\left(a_{1 \mu}+a_{3 \mu}\right)-\left(a_{2 \mu}+a_{4 \mu}\right)\right) / 4$.

Proposition 57. Let $A_{I}=\left\langle\left(a_{1 \mu}, a_{2 \mu}, a_{3 \mu}\right),\left(a_{1 v}^{\prime}, a_{2 v}^{\prime}, a_{3 \nu}^{\prime}\right)\right\rangle$ be a triangular intuitionistic fuzzy number. Then $J_{5}\left(A_{I}\right)=\left(-\left(a_{3 \mu}-\right.\right.$ $\left.\left.a_{1 \mu}\right)-\left(a_{3 \nu}^{\prime}-a_{1 \nu}^{\prime}\right)\right) / 8$.

Proposition 58. Let $A_{I}=\left(\left[a_{1 \mu}, a_{2 \mu}\right],\left[a_{1 v}^{\prime}, a_{2 \gamma}^{\prime}\right]\right)$ be an interval valued intuitionistic fuzzy number. Then $J_{5}\left(A_{I}\right)=0$.

Theorem 59. Let $A_{I}, B_{I} \in C_{5}$. If $A_{I} \triangleleft B_{I}$ then $J_{5}\left(A_{I}\right)>$ $J_{5}\left(B_{I}\right)$.

Proof. Let us assume that $A_{I} \triangleleft B_{I}$. We claim that $J_{5}\left(A_{I}\right)-$ $J_{5}\left(B_{I}\right)>0$.

By Definition 53, we have

$$
\begin{aligned}
& a_{1 \mu} \geq b_{1 \mu}, \\
& a_{2 \mu} \leq b_{2 \mu}, \\
& a_{3 \mu} \geq b_{3 \mu}, \\
& a_{4 \mu} \leq b_{4 \mu}, \\
& a_{1 v}^{\prime} \geq b_{1 v}^{\prime}, \\
& a_{2 v}^{\prime} \leq b_{2 v}^{\prime}, \\
& a_{3 v}^{\prime} \geq b_{3 v}^{\prime}, \\
& a_{4 v}^{\prime} \leq b_{4 v}^{\prime} .
\end{aligned}
$$

Now,

$$
\begin{gathered}
8\left(J_{5}\left(A_{I}\right)-J_{5}\left(B_{I}\right)\right)=\left[\left(a_{1 \mu}-b_{1 \mu}\right)+\left(b_{2 \mu}-a_{2 \mu}\right)\right. \\
\left.+\left(a_{3 \mu}-b_{3 \mu}\right)+\left(b_{4 \mu}-a_{4 \mu}\right)\right]+\left[\left(a_{1 v}^{\prime}-b_{1 v}^{\prime}\right)\right. \\
\left.+\left(b_{2 v}^{\prime}-a_{2 v}^{\prime}\right)+\left(a_{3 v}^{\prime}-b_{3 v}^{\prime}\right)+\left(b_{4 v}^{\prime}-a_{4 v}^{\prime}\right)\right] .
\end{gathered}
$$

From (35) it is very easy to see that all the terms in (36) are positive. Therefore their sum is also positive. From Definition 53, we know that at least one of the above inequalities in (35) becomes strict inequality and hence we get $J_{5}\left(A_{I}\right)$ $J_{5}\left(B_{I}\right)>0$.

Theorem 60. Let $A_{I}, B_{I} \in C_{5}$. If $J_{5}\left(A_{I}\right)=J_{5}\left(B_{I}\right)$ then $A_{I}=$ $B_{I}$.

Proof. Let us assume $J_{5}\left(A_{I}\right)=J_{5}\left(B_{I}\right)$. We claim that $A_{I}=B_{I}$. By Definition 53, without loss of generality, we have

$$
\begin{aligned}
& a_{1 \mu} \geq b_{1 \mu}, \\
& a_{2 \mu} \leq b_{2 \mu}, \\
& a_{3 \mu} \geq b_{3 \mu}, \\
& a_{4 \mu} \leq b_{4 \mu}, \\
& a_{1 v}^{\prime} \geq b_{1 v}^{\prime}, \\
& a_{2 v}^{\prime} \leq b_{2 v}^{\prime}, \\
& a_{3 v}^{\prime} \geq b_{3 v}^{\prime}, \\
& a_{4 v}^{\prime} \leq b_{4 v}^{\prime} .
\end{aligned}
$$

Now,

$$
\begin{gathered}
8\left(J_{5}\left(A_{I}\right)-J_{5}\left(B_{I}\right)\right)=\left[\left(a_{1 \mu}-b_{1 \mu}\right)+\left(b_{2 \mu}-a_{2 \mu}\right)\right. \\
\left.\quad+\left(a_{3 \mu}-b_{3 \mu}\right)+\left(b_{4 \mu}-a_{4 \mu}\right)\right]+\left[\left(a_{1 \nu}^{\prime}-b_{1 v}^{\prime}\right)\right. \\
\left.\quad+\left(b_{2 \nu}^{\prime}-a_{2 \nu}^{\prime}\right)+\left(a_{3 \nu}^{\prime}-b_{3 \nu}^{\prime}\right)+\left(b_{4 \nu}^{\prime}-a_{4 v}^{\prime}\right)\right]=0 .
\end{gathered}
$$

Therefore from (37) and (38), it is clear that all the terms in (38) are positive and therefore their sum gives zero only when each term is equal to zero. Hence $a_{1 \mu}=b_{1 \mu}, a_{2 \mu}=b_{2 \mu}, a_{3 \mu}=$ $b_{3 \mu}, a_{4 \mu}=b_{4 \mu}$ and $a_{1 v}^{\prime}=b_{1 v}^{\prime}, a_{2 v}^{\prime}=b_{2 v}^{\prime}, a_{3 v}^{\prime}=b_{3 v}^{\prime}, a_{4 v}^{\prime}=b_{4 v}^{\prime}$. Hence $A_{I}=B_{I}$.

Note 10. The spread score can be calculated to any TrIFN. But $J_{5}$ gives total order on $C_{5}$ which is seen from Theorems 59 and 60.

Definition 61. If $J_{5}\left(A_{I}\right)>J_{5}\left(B_{I}\right)\left(J_{5}\left(A_{I}\right)<J_{5}\left(B_{I}\right)\right)$ then $A_{I}>$ $B_{I}\left(A_{I}<B_{I}\right)$.

The following example is used to explain the ranking procedure defined on $C_{5}$. 
Example 62. Let $A_{I}=\langle(0.15,0.23,0.31,0.45),(0.1,0.16$, $0.35,0.5)\rangle$ and $B_{I}=\langle(0.1,0.25,0.25,0.5),(0,0.2,0.3,0.6)\rangle \epsilon$ $C_{5}$. Now $J_{5}\left(A_{I}\right)=-0.05375$ and $J_{5}\left(B_{I}\right)=-0.1125, J_{5}\left(A_{I}\right)>$ $J_{5}\left(B_{I}\right)$.

Hence $A_{I}>B_{I}$.

Example 63. Let $A_{I}=\left\langle\left(a_{1 \mu}, a_{2 \mu}, a_{3 \mu}, a_{4 \mu}\right),\left(a_{1 v}^{\prime}, a_{2 v}^{\prime}, a_{3 v}^{\prime}, a_{4 v}^{\prime}\right)\right\rangle$ and $B_{I}=\left\langle\left(a_{1 \mu}+\epsilon, a_{2 \mu}-\epsilon, a_{3 \mu}+\epsilon, a_{4 \mu}-\epsilon\right),\left(a_{1 \nu}^{\prime}+\epsilon, a_{2 v}^{\prime}-\right.\right.$ $\left.\left.\epsilon, a_{3 \nu}^{\prime}+\epsilon, a_{4 v}^{\prime}-\epsilon\right)\right\rangle \in \operatorname{TrIFN}$ and $\epsilon \in[0,1]$.

Now

$$
\begin{aligned}
& J_{1}\left(A_{I}\right)=J_{1}\left(B_{I}\right) \\
& =\frac{\left(a_{1 \mu}+a_{2 \mu}\right)-\left(a_{3 \mu}+a_{4 \mu}\right)+\left(a_{1 \nu}^{\prime}+a_{2 \nu}^{\prime}\right)-\left(a_{3 \nu}^{\prime}+a_{4 \nu}^{\prime}\right)}{8}, \\
& J_{2}\left(A_{I}\right)=J_{2}\left(B_{I}\right) \\
& =\frac{\left(a_{1 \mu}+a_{2 \mu}\right)-\left(a_{3 \mu}+a_{4 \mu}\right)-\left(a_{1 \nu}^{\prime}+a_{2 \nu}^{\prime}\right)+\left(a_{3 \nu}^{\prime}+a_{4 \nu}^{\prime}\right)}{8}, \\
& J_{3}\left(A_{I}\right)=J_{3}\left(B_{I}\right) \\
& =\frac{\left(a_{1 \nu}^{\prime}+a_{2 \nu}^{\prime}+a_{3 \nu}^{\prime}+a_{4 \nu}^{\prime}\right)-\left(a_{1 \mu}+a_{2 \mu}+a_{3 \mu}+a_{4 \mu}\right)}{8}, \\
& J_{4}\left(A_{I}\right)=J_{4}\left(B_{I}\right) \\
& =\frac{\left(a_{1 \mu}+a_{2 \mu}+a_{3 \mu}+a_{4 \mu}\right)+\left(a_{1 \nu}^{\prime}+a_{2 \nu}^{\prime}+a_{3 \nu}^{\prime}+a_{4 \nu}^{\prime}\right)}{8} .
\end{aligned}
$$

But

$$
\begin{aligned}
& J_{5}\left(A_{I}\right) \\
& =\frac{\left(a_{1 \mu}+a_{3 \mu}\right)-\left(a_{2 \mu}+a_{4 \mu}\right)+\left(a_{1 \nu}^{\prime}+a_{3 \nu}^{\prime}\right)-\left(a_{2 \nu}^{\prime}+a_{4 v}^{\prime}\right)}{8}, \\
& J_{5}\left(B_{I}\right) \\
& =\frac{\left(a_{1 \mu}+a_{3 \mu}\right)-\left(a_{2 \mu}+a_{4 \mu}\right)+\left(a_{1 \nu}^{\prime}+a_{3 \nu}^{\prime}\right)-\left(a_{2 v}^{\prime}+a_{4 v}^{\prime}\right)}{8} \\
& \quad+\epsilon \Longrightarrow \\
& J_{5}\left(B_{I}\right)>J_{5}\left(A_{I}\right) .
\end{aligned}
$$

Hence $B_{I}>A_{I}$. In this example the importance of $J_{5}$ in ranking arbitrary TrIFNs is shown.

The inability of $J_{5}$ in comparing any two TrIFNs is shown in the following example.

Example 64. Let $A_{I}=\left\langle\left(a_{1 \mu}, a_{2 \mu}, a_{3 \mu}, a_{4 \mu}\right),\left(a_{1 \nu}^{\prime}, a_{2 v}^{\prime}, a_{3 \nu}^{\prime}, a_{4 v}^{\prime}\right)\right\rangle$ and $B_{I}=\left\langle\left(a_{1 \mu}+\epsilon, a_{2 \mu}-\epsilon, a_{3 \mu}+\epsilon, a_{4 \mu}-\epsilon\right),\left(a_{1 \nu}^{\prime}-\epsilon, a_{2 \nu}^{\prime}+\epsilon, a_{3 \nu}^{\prime}-\right.\right.$ $\left.\left.\epsilon, a_{4 v}^{\prime}+\epsilon\right)\right\rangle \in \operatorname{TrIFN}$ and $\epsilon \in[0,1]$ but not in $C_{5}$.

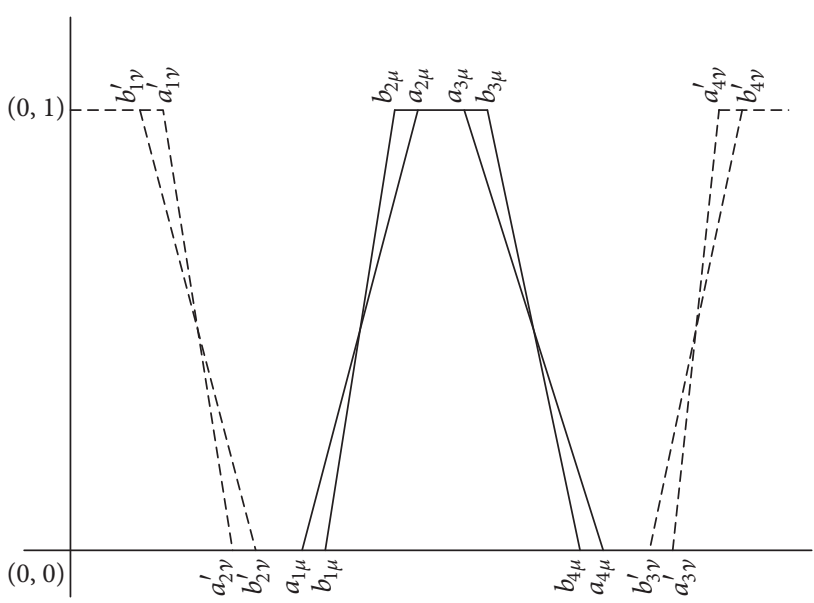

FIgURE 7: Pictorial representation of Example 64.

Now

$$
\begin{aligned}
& J_{1}\left(A_{I}\right)=J_{1}\left(B_{I}\right) \\
& =\frac{\left(a_{1 \mu}+a_{2 \mu}\right)-\left(a_{3 \mu}+a_{4 \mu}\right)+\left(a_{1 \nu}^{\prime}+a_{2 \nu}^{\prime}\right)-\left(a_{3 \nu}^{\prime}+a_{4 \nu}^{\prime}\right)}{8}, \\
& J_{2}\left(A_{I}\right)=J_{2}\left(B_{I}\right) \\
& =\frac{\left(a_{1 \mu}+a_{2 \mu}\right)-\left(a_{3 \mu}+a_{4 \mu}\right)-\left(a_{1 \nu}^{\prime}+a_{2 \nu}^{\prime}\right)+\left(a_{3 \nu}^{\prime}+a_{4 \nu}^{\prime}\right)}{8}, \\
& J_{3}\left(A_{I}\right)=J_{3}\left(B_{I}\right) \\
& =\frac{\left(a_{1 \nu}^{\prime}+a_{2 \nu}^{\prime}+a_{3 \nu}^{\prime}+a_{4 v}^{\prime}\right)-\left(a_{1 \mu}+a_{2 \mu}+a_{3 \mu}+a_{4 \mu}\right)}{8}, \\
& J_{4}\left(A_{I}\right)=J_{4}\left(B_{I}\right) \\
& =\frac{\left(a_{1 \mu}+a_{2 \mu}+a_{3 \mu}+a_{4 \mu}\right)+\left(a_{1 \nu}^{\prime}+a_{2 \nu}^{\prime}+a_{3 \nu}^{\prime}+a_{4 \nu}^{\prime}\right)}{8}, \\
& J_{5}\left(A_{I}\right)=J_{5}\left(B_{I}\right) \\
& =\frac{\left(a_{1 \mu}+a_{3 \mu}\right)-\left(a_{2 \mu}+a_{4 \mu}\right)+\left(a_{1 \nu}^{\prime}+a_{3 \nu}^{\prime}\right)-\left(a_{2 \nu}^{\prime}+a_{4 v}^{\prime}\right)}{8} .
\end{aligned}
$$

But $A_{I} \neq B_{I}$.

Pictorial representation of this example is given in Figure 7.

Example 64 shows that all the above defined scores are not enough to cover the entire class of TrIFNs; therefore we are introducing another score function in the next subsection.

3.6. Nonaccuracy Score of a Trapezoidal Intuitionistic Fuzzy Number. In this subsection, a new subclass of TrIFNs is introduced. The nonaccuracy score function on this class of trapezoidal intuitionistic fuzzy numbers is defined and some of its properties are studied using illustrative examples. 
Definition 65. Let $A_{I}$ and $B_{I}$ be two TrIFNs.

A special subclass $C_{6}$ of the set of TrIFNs consist of TrIFNs for which every pair of $A_{I}$ and $B_{I}$ are with $a_{1 \mu} \geq b_{1 \mu}, a_{2 \mu} \leq$ $b_{2 \mu}, a_{3 \mu} \geq b_{3 \mu}, a_{4 \mu} \leq b_{4 \mu}$ and $a_{1 v}^{\prime} \leq b_{1 v}^{\prime}, a_{2 v}^{\prime} \geq b_{2 v}^{\prime}, a_{3 v}^{\prime} \leq$ $b_{3 v}^{\prime}, a_{4 v}^{\prime} \geq b_{4 v}^{\prime}$. The exact relation on $C_{6}$ is denoted as $\unrhd$ and defined as follows: if $A_{I}, B_{I} \in C_{6} \subset \operatorname{TrIFN}$ such that $A_{I} \unrhd B_{I}$ then $a_{1 \mu} \geq b_{1 \mu}, a_{2 \mu} \leq b_{2 \mu}, a_{3 \mu} \geq b_{3 \mu}, a_{4 \mu} \leq b_{4 \mu}$ and $a_{1 \nu}^{\prime} \leq$ $b_{1 v}^{\prime}, a_{2 v}^{\prime} \geq b_{2 v}^{\prime}, a_{3 v}^{\prime} \leq b_{3 v}^{\prime}, a_{4 v}^{\prime} \geq b_{4 v}^{\prime}$. If $A_{I} \triangleright B_{I}$ then one of the above inequalities becomes strict inequality.

Note 11. By Definition 65, we note that any pair of members of $C_{6}$ are related under $\triangleright$.

The score function which measures the nonaccuracy is defined as follows.

Definition 66. Let $A_{I}$ be a trapezoidal intuitionistic fuzzy number. Then the nonaccuracy score of $A_{I}$ is defined by

$$
\begin{aligned}
& J_{6}\left(A_{I}\right) \\
& =\frac{\left(a_{2 \mu}+a_{4 \mu}\right)-\left(a_{1 \mu}+a_{3 \mu}\right)+\left(a_{1 \nu}^{\prime}+a_{3 \nu}^{\prime}\right)-\left(a_{2 \nu}^{\prime}+a_{4 \nu}^{\prime}\right)}{8} .
\end{aligned}
$$

The proofs of the following propositions are easy and hence they are omitted.

Proposition 67. For any real number $r \in[0,1], J_{6}(r)=0$.

Proposition 68. If $A_{I}=\left(a_{1 \mu}, a_{2 \mu}, a_{3 \mu}, a_{4 \mu}\right)$ is a trapezoidal fuzzy number, then $J_{6}\left(A_{I}\right)=0$.

Proposition 69. Let $A_{I}=\left\langle\left(a_{1 \mu}, a_{2 \mu}, a_{3 \mu}\right),\left(a_{1 v}^{\prime}, a_{2 v}^{\prime}, a_{3 v}^{\prime}\right)\right\rangle$ be a triangular intuitionistic fuzzy number. Then $J_{6}\left(A_{I}\right)=\left(\left(a_{1 v}^{\prime}-\right.\right.$ $\left.\left.a_{3 \nu}^{\prime}\right)-\left(a_{1 \mu}-a_{3 \mu}\right)\right) / 8$.

Proposition 70. Let $A_{I}=\left(\left[a_{1 \mu}, a_{2 \mu}\right],\left[a_{1 v}^{\prime}, a_{2 \gamma}^{\prime}\right]\right)$ be an interval valued intuitionistic fuzzy number. Then $J_{6}\left(A_{I}\right)=0$.

Note 12. The nonaccuracy score can be calculated to any TrIFN. But $J_{6}$ gives total order on $C_{6}$ which is proved in Theorems 71 and 72 .

Theorem 71. Let $A_{I}, B_{I} \in C_{6}$. If $A_{I} \triangleright B_{I}$ then $J_{6}\left(A_{I}\right)<$ $J_{6}\left(B_{I}\right)$.

Proof. Let us assume that $A_{I} \triangleright B_{I}$. We claim that $J_{6}\left(B_{I}\right)-$ $J_{6}\left(A_{I}\right)>0$.

By Definition 65, we have

$$
\begin{aligned}
& a_{1 \mu} \geq b_{1 \mu}, \\
& a_{2 \mu} \leq b_{2 \mu}, \\
& a_{3 \mu} \geq b_{3 \mu}, \\
& a_{4 \mu} \leq b_{4 \mu}, \\
& a_{1 \nu}^{\prime} \leq b_{1 \nu}^{\prime}, \\
& a_{2 \nu}^{\prime} \geq b_{2 \nu}^{\prime},
\end{aligned}
$$

$$
\begin{aligned}
& a_{3 v}^{\prime} \leq b_{3 v}^{\prime}, \\
& a_{4 v}^{\prime} \geq b_{4 v}^{\prime}
\end{aligned}
$$

and at least one of these inequalities becomes strict.

Now,

$$
\begin{gathered}
8\left(J_{6}\left(B_{I}\right)-J_{6}\left(A_{I}\right)\right)=\left[\left(a_{1 \mu}-b_{1 \mu}\right)+\left(b_{2 \mu}-a_{2 \mu}\right)\right. \\
\left.+\left(a_{3 \mu}-b_{3 \mu}\right)+\left(b_{4 \mu}-a_{4 \mu}\right)\right]+\left[\left(b_{1 v}^{\prime}-a_{1 v}^{\prime}\right)\right. \\
\left.+\left(a_{2 v}^{\prime}-b_{2 v}^{\prime}\right)+\left(b_{3 v}^{\prime}-a_{3 v}^{\prime}\right)+\left(a_{4 v}^{\prime}-b_{4 v}^{\prime}\right)\right] .
\end{gathered}
$$

From (43) it is very easy to see that all the terms in (44) are positive and therefore their sum is also positive. From Definition 65, we know that at least one of the above inequalities in (43) becomes strict inequality and hence we get $J_{6}\left(B_{I}\right)-J_{6}\left(A_{I}\right)>0$.

Theorem 72. Let $A_{I}, B_{I} \in C_{6}$. If $J_{6}\left(A_{I}\right)=J_{6}\left(B_{I}\right)$ then $A_{I}=$ $B_{I}$.

Proof. Let us assume $J_{6}\left(A_{I}\right)=J_{6}\left(B_{I}\right)$. We claim that $A_{I}=B_{I}$.

By Definition 65, without loss of generality, we have

$$
\begin{aligned}
& a_{1 \mu} \geq b_{1 \mu}, \\
& a_{2 \mu} \leq b_{2 \mu}, \\
& a_{3 \mu} \geq b_{3 \mu}, \\
& a_{4 \mu} \leq b_{4 \mu}, \\
& a_{1 v}^{\prime} \leq b_{1 v}^{\prime}, \\
& a_{2 v}^{\prime} \geq b_{2 v}^{\prime}, \\
& a_{3 v}^{\prime} \leq b_{3 v}^{\prime}, \\
& a_{4 v}^{\prime} \geq b_{4 v}^{\prime} .
\end{aligned}
$$

Now,

$$
\begin{aligned}
& 8\left(J_{6}\left(B_{I}\right)-J_{6}\left(A_{I}\right)\right)=\left[\left(a_{1 \mu}-b_{1 \mu}\right)+\left(b_{2 \mu}-a_{2 \mu}\right)\right. \\
& \left.\quad+\left(a_{3 \mu}-b_{3 \mu}\right)+\left(b_{4 \mu}-a_{4 \mu}\right)\right]+\left[\left(b_{1 v}^{\prime}-a_{1 v}^{\prime}\right)\right. \\
& \left.\quad+\left(a_{2 v}^{\prime}-b_{2 v}^{\prime}\right)+\left(b_{3 \nu}^{\prime}-a_{3 \nu}^{\prime}\right)+\left(a_{4 v}^{\prime}-b_{4 v}^{\prime}\right)\right]=0 .
\end{aligned}
$$

Therefore from (45) and (46), it is clear that all the terms in (46) are positive and therefore their sum gives zero only when each term is equal to zero. Hence $a_{1 \mu}=b_{1 \mu}, a_{2 \mu}=b_{2 \mu}, a_{3 \mu}=$ $b_{3 \mu}, a_{4 \mu}=b_{4 \mu}$ and $a_{1 v}^{\prime}=b_{1 v}^{\prime}, a_{2 v}^{\prime}=b_{2 v}^{\prime}, a_{3 v}^{\prime}=b_{3 v}^{\prime}, a_{4 v}^{\prime}=b_{4 v}^{\prime}$. Hence $A_{I}=B_{I}$.

Definition 73. If $J_{6}\left(A_{I}\right)>J_{6}\left(B_{I}\right)\left(J_{6}\left(A_{I}\right)<J_{6}\left(B_{I}\right)\right)$, then $A_{I}>$ $B_{I}\left(A_{I}<B_{I}\right)$.

Ranking procedure introduced in Definition 73 is explained in Example 74. 
Example 74. Let $A_{I}=\langle(0.23,0.27,0.34,0.51),(0.17,0.23$, $0.4,0.6)\rangle$ and $B_{I}=\langle(0.2,0.3,0.3,0.55),(0.2,0.2,0.5,0.55)\rangle \epsilon$ $C_{6}$. Now $J_{6}\left(A_{I}\right)=-0.00625$ and $J_{6}\left(B_{I}\right)=0.0375, J_{6}\left(A_{I}\right)<$ $J_{6}\left(B_{I}\right)$.

Hence $A_{I}<B_{I}$.

The efficiency of $J_{6}$ in ranking TrIFNs is shown in the following example.

Example 75. Let $A_{I}=\left\langle\left(a_{1 \mu}, a_{2 \mu}, a_{3 \mu}, a_{4 \mu}\right),\left(a_{1 v}^{\prime}, a_{2 v}^{\prime}, a_{3 v}^{\prime}, a_{4 \nu}^{\prime}\right)\right\rangle$ and $B_{I}=\left\langle\left(a_{1 \mu}+\epsilon, a_{2 \mu}-\epsilon, a_{3 \mu}+\epsilon, a_{4 \mu}-\epsilon\right),\left(a_{1 \nu}^{\prime}-\epsilon, a_{2 \nu}^{\prime}+\right.\right.$ $\left.\left.\epsilon, a_{3 \nu}^{\prime}-\epsilon, a_{4 v}^{\prime}+\epsilon\right)\right\rangle \in \operatorname{TrIFN}$ and $\epsilon \in[0,1]$.

Now

$$
\begin{aligned}
& J_{1}\left(A_{I}\right)=J_{1}\left(B_{I}\right) \\
& =\frac{\left(a_{1 \mu}+a_{2 \mu}\right)-\left(a_{3 \mu}+a_{4 \mu}\right)+\left(a_{1 \nu}^{\prime}+a_{2 \nu}^{\prime}\right)-\left(a_{3 \nu}^{\prime}+a_{4 \nu}^{\prime}\right)}{8}, \\
& J_{2}\left(A_{I}\right)=J_{2}\left(B_{I}\right) \\
& =\frac{\left(a_{1 \mu}+a_{2 \mu}\right)-\left(a_{3 \mu}+a_{4 \mu}\right)-\left(a_{1 \nu}^{\prime}+a_{2 \nu}^{\prime}\right)+\left(a_{3 \nu}^{\prime}+a_{4 \nu}^{\prime}\right)}{8}, \\
& J_{3}\left(A_{I}\right)=J_{3}\left(B_{I}\right) \\
& =\frac{\left(a_{1 \nu}^{\prime}+a_{2 \nu}^{\prime}+a_{3 \nu}^{\prime}+a_{4 \nu}^{\prime}\right)-\left(a_{1 \mu}+a_{2 \mu}+a_{3 \mu}+a_{4 \mu}\right)}{8}, \\
& J_{4}\left(A_{I}\right)=J_{4}\left(B_{I}\right) \\
& =\frac{\left(a_{1 \mu}+a_{2 \mu}+a_{3 \mu}+a_{4 \mu}\right)+\left(a_{1 \nu}^{\prime}+a_{2 \nu}^{\prime}+a_{3 \nu}^{\prime}+a_{4 \nu}^{\prime}\right)}{8}, \\
& J_{5}\left(A_{I}\right)=J_{5}\left(B_{I}\right) \\
& =\frac{\left(a_{1 \mu}+a_{3 \mu}\right)-\left(a_{2 \mu}+a_{4 \mu}\right)+\left(a_{1 \nu}^{\prime}+a_{3 \nu}^{\prime}\right)-\left(a_{2 \nu}^{\prime}+a_{4 \nu}^{\prime}\right)}{8} .
\end{aligned}
$$

But

$$
\begin{aligned}
& J_{6}\left(A_{I}\right) \\
& =\frac{\left(a_{2 \mu}+a_{4 \mu}\right)-\left(a_{1 \mu}+a_{3 \mu}\right)+\left(a_{1 v}^{\prime}+a_{3 v}^{\prime}\right)-\left(a_{2 v}^{\prime}+a_{4 v}^{\prime}\right)}{8}, \\
& J_{6}\left(B_{I}\right) \\
& =\frac{\left(a_{2 \mu}+a_{4 \mu}\right)-\left(a_{1 \mu}+a_{3 \mu}\right)+\left(a_{1 v}^{\prime}+a_{3 \nu}^{\prime}\right)-\left(a_{2 v}^{\prime}+a_{4 v}^{\prime}\right)}{8} \\
& \quad+\epsilon \Longrightarrow \\
& J_{6}\left(A_{I}\right)<J_{6}\left(B_{I}\right) .
\end{aligned}
$$

Hence $A_{I}<B_{I}$.

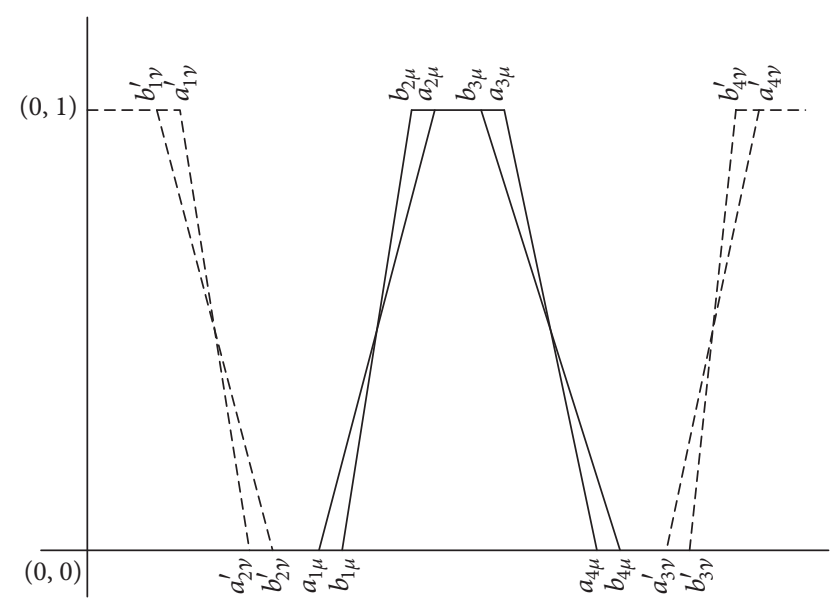

FIGURE 8: Pictorial representation of Example 76.

Example 76. Let $A_{I}=\left\langle\left(a_{1 \mu}, a_{2 \mu}, a_{3 \mu}, a_{4 \mu}\right),\left(a_{1 v}^{\prime}, a_{2 v}^{\prime}, a_{3 v}^{\prime}, a_{4 v}^{\prime}\right)\right\rangle$ and $B_{I}=\left\langle\left(a_{1 \mu}+\epsilon, a_{2 \mu}-\epsilon, a_{3 \mu}-\epsilon, a_{4 \mu}+\epsilon\right),\left(a_{1 v}^{\prime}-\epsilon, a_{2 v}^{\prime}+\right.\right.$ $\left.\left.\epsilon, a_{3 \nu}^{\prime}+\epsilon, a_{4 v}^{\prime}-\epsilon\right)\right\rangle \in \operatorname{TrIFN}$ and $\epsilon \in[0,1]$ but not in $C_{6}$, where $\epsilon \in[0,1]$. Now

$$
\begin{aligned}
& J_{1}\left(A_{I}\right)=J_{1}\left(B_{I}\right) \\
& =\frac{\left(a_{1 \mu}+a_{2 \mu}\right)-\left(a_{3 \mu}+a_{4 \mu}\right)+\left(a_{1 \nu}^{\prime}+a_{2 \nu}^{\prime}\right)-\left(a_{3 \nu}^{\prime}+a_{4 \nu}^{\prime}\right)}{8}, \\
& J_{2}\left(A_{I}\right)=J_{2}\left(B_{I}\right) \\
& =\frac{\left(a_{1 \mu}+a_{2 \mu}\right)-\left(a_{3 \mu}+a_{4 \mu}\right)-\left(a_{1 \nu}^{\prime}+a_{2 v}^{\prime}\right)+\left(a_{3 \nu}^{\prime}+a_{4 v}^{\prime}\right)}{8}, \\
& J_{3}\left(A_{I}\right)=J_{3}\left(B_{I}\right) \\
& =\frac{\left(a_{1 \nu}^{\prime}+a_{2 v}^{\prime}+a_{3 \nu}^{\prime}+a_{4 \nu}^{\prime}\right)-\left(a_{1 \mu}+a_{2 \mu}+a_{3 \mu}+a_{4 \mu}\right)}{8}, \\
& J_{4}\left(A_{I}\right)=J_{4}\left(B_{I}\right) \\
& =\frac{\left(a_{1 \mu}+a_{2 \mu}+a_{3 \mu}+a_{4 \mu}\right)+\left(a_{1 \nu}^{\prime}+a_{2 \nu}^{\prime}+a_{3 \nu}^{\prime}+a_{4 \nu}^{\prime}\right)}{8}, \\
& J_{5}\left(A_{I}\right)=J_{5}\left(B_{I}\right) \\
& =\frac{\left(a_{1 \mu}+a_{3 \mu}\right)-\left(a_{2 \mu}+a_{4 \mu}\right)+\left(a_{1 \nu}^{\prime}+a_{3 \nu}^{\prime}\right)-\left(a_{2 \nu}^{\prime}+a_{4 \nu}^{\prime}\right)}{8} \text {. }
\end{aligned}
$$

But

$$
\begin{aligned}
& J_{6}\left(A_{I}\right)=J_{6}\left(B_{I}\right) \\
& =\frac{\left(a_{2 \mu}+a_{4 \mu}\right)-\left(a_{1 \mu}+a_{3 \mu}\right)+\left(a_{1 \nu}^{\prime}+a_{3 \nu}^{\prime}\right)-\left(a_{2 \nu}^{\prime}+a_{4 \nu}^{\prime}\right)}{8} .
\end{aligned}
$$

But $A_{I} \neq B_{I}$. ure 8 .

Pictorial representation of this example is given in Fig-

Example 76 shows that the score functions $J_{1}$ to $J_{6}$ are not sufficient to cover the entire class of TrIFNs and hence 
the class of TrIFNs in the above example excite us to define new score function that can cover a subclass of TrIFNs which cannot be covered by $J_{1}$ to $J_{6}$.

3.7. Left Area of a Trapezoidal Intuitionistic Fuzzy Number. In this subsection, another subclass of TrIFNs is introduced and the left area score function on this class of trapezoidal intuitionistic fuzzy numbers is defined and some of its properties are studied using illustrative examples.

Definition 77. Let $A_{I}$ and $B_{I}$ be two TrIFNs.

A special subclass $C_{7}$ of the set of TrIFNs consist of TrIFNs for which every pair of $A_{I}$ and $B_{I}$ are with $a_{1 \mu} \leq b_{1 \mu}, a_{2 \mu} \geq$ $b_{2 \mu}, a_{3 \mu} \geq b_{3 \mu}, a_{4 \mu} \leq b_{4 \mu}$ and $a_{1 v}^{\prime} \geq b_{1 v}^{\prime}, a_{2 v}^{\prime} \leq b_{2 v}^{\prime}, a_{3 v}^{\prime} \leq$ $b_{3 v}^{\prime}, a_{4 v}^{\prime} \geq b_{4 v}^{\prime}$. The nonaccurate relation on $C_{7}$ is denoted as $\ll$ and defined as follows: if $A_{I}, B_{I} \in C_{7} \subset$ TrIFN such that $\bar{A}_{I} \ll B_{I}$ then $a_{1 \mu} \leq b_{1 \mu}, a_{2 \mu} \geq b_{2 \mu}, a_{3 \mu} \geq b_{3 \mu}, a_{4 \mu} \leq b_{4 \mu}$ and $a_{1 v}^{\prime} \geq b_{1 v}^{\prime}, a_{2 v}^{\prime} \leq b_{2 v}^{\prime}, a_{3 v}^{\prime} \leq b_{3 v}^{\prime}, a_{4 v}^{\prime} \geq b_{4 v}^{\prime}$.

If $A_{I} \ll B_{I}$ then one of the above inequalities becomes strict inequality.

Note 13. By Definition 77, we note that any pair of members of $C_{7}$ are related under $\ll$.

The score function which measures the left area is defined as follows.

Definition 78. Let $A_{I}$ be a trapezoidal intuitionistic fuzzy number. Then the left area of $A_{I}$ is defined by

$$
\begin{aligned}
& J_{7}\left(A_{I}\right) \\
& =\frac{\left(a_{3 \nu}^{\prime}-a_{1 \nu}^{\prime}\right)+\left(a_{2 \nu}^{\prime}-a_{4 \nu}^{\prime}\right)-\left(a_{3 \mu}-a_{1 \mu}\right)-\left(a_{2 \mu}-a_{4 \mu}\right)}{8} .
\end{aligned}
$$

The proofs of the following propositions are immediate application of Definition 78 and hence they are omitted.

Proposition 79. For any real number $r \in[0,1], J_{7}(r)=0$.

Proposition 80. If $A_{I}=\left(a_{1 \mu}, a_{2 \mu}, a_{3 \mu}, a_{4 \mu}\right)$ is a trapezoidal fuzzy number, then $J_{7}\left(A_{I}\right)=0$.

Proposition 81. Let $A_{I}=\left\langle\left(a_{1 \mu}, a_{2 \mu}, a_{3 \mu}\right),\left(a_{1 v}^{\prime}, a_{2 v}^{\prime}, a_{3 v}^{\prime}\right)\right\rangle$ be a triangular intuitionistic fuzzy number. Then $J_{7}\left(A_{I}\right)=\left(\left(a_{1 \mu}+\right.\right.$ $\left.\left.a_{3 \mu}-2 a_{2 \mu}\right)+\left(2 a_{2 v}^{\prime}-a_{1 v}^{\prime}-a_{3 v}^{\prime}\right)\right) / 8$.

Proposition 82. Let $A_{I}=\left(\left[a_{1 \mu}, a_{2 \mu}\right],\left[a_{1 v}^{\prime}, a_{2 \gamma}^{\prime}\right]\right)$ be an interval valued intuitionistic fuzzy number. Then $J_{7}\left(A_{I}\right)=0$.

Theorem 83. Let $A_{I}, B_{I} \in C_{7}$. If $A_{I} \ll B_{I}$ then $J_{7}\left(A_{I}\right)<$ $J_{7}\left(B_{I}\right)$.

Proof. Let us assume that $A_{I} \ll B_{I}$.

We claim that $J_{7}\left(B_{I}\right)-J_{7}\left(A_{I}\right)>0$.

By Definition 77, we have

$$
\begin{aligned}
& a_{1 \mu} \leq b_{1 \mu}, \\
& a_{2 \mu} \geq b_{2 \mu},
\end{aligned}
$$

$$
\begin{aligned}
& a_{3 \mu} \geq b_{3 \mu}, \\
& a_{4 \mu} \leq b_{4 \mu}, \\
& a_{1 v}^{\prime} \geq b_{1 v}^{\prime}, \\
& a_{2 v}^{\prime} \leq b_{2 v}^{\prime}, \\
& a_{3 v}^{\prime} \leq b_{3 v}^{\prime}, \\
& a_{4 v}^{\prime} \geq b_{4 v}^{\prime} .
\end{aligned}
$$

Now,

$$
\begin{gathered}
8\left(J_{7}\left(B_{I}\right)-J_{7}\left(A_{I}\right)\right)=\left[\left(b_{1 \mu}-a_{1 \mu}\right)+\left(a_{2 \mu}-b_{2 \mu}\right)\right. \\
\left.+\left(a_{3 \mu}-b_{3 \mu}\right)+\left(b_{4 \mu}-a_{4 \mu}\right)\right]+\left[\left(a_{1 v}^{\prime}-b_{1 v}^{\prime}\right)\right. \\
\left.+\left(b_{2 v}^{\prime}-a_{2 v}^{\prime}\right)+\left(b_{3 \nu}^{\prime}-a_{3 \nu}^{\prime}\right)+\left(a_{4 v}^{\prime}-b_{4 v}^{\prime}\right)\right] .
\end{gathered}
$$

From (52) it is very easy to see that all the terms in (53) are positive. Therefore their sum is also positive. From Definition 77, we know that at least one of the above inequalities in $(52)$ becomes strict inequality and hence we get $J_{7}\left(B_{I}\right)$ $J_{7}\left(A_{I}\right)>0$, hence the proof.

Theorem 84. Let $A_{I}, B_{I} \in C_{7}$. If $J_{7}\left(A_{I}\right)=J_{7}\left(B_{I}\right)$ then $A_{I}=$ $B_{I}$.

Proof. Let us assume $J_{7}\left(A_{I}\right)=J_{7}\left(B_{I}\right)$. We claim that $A_{I}=B_{I}$. By Definition 77, without loss of generality, we have

$$
\begin{aligned}
& a_{1 \mu} \leq b_{1 \mu}, \\
& a_{2 \mu} \geq b_{2 \mu}, \\
& a_{3 \mu} \geq b_{3 \mu}, \\
& a_{4 \mu} \leq b_{4 \mu}, \\
& a_{1 v}^{\prime} \geq b_{1 v}^{\prime}, \\
& a_{2 v}^{\prime} \leq b_{2 v}^{\prime}, \\
& a_{3 v}^{\prime} \leq b_{3 v}^{\prime}, \\
& a_{4 v}^{\prime} \geq b_{4 v}^{\prime} .
\end{aligned}
$$

Now,

$$
\begin{gathered}
8\left(J_{7}\left(B_{I}\right)-J_{7}\left(A_{I}\right)\right)=\left[\left(b_{1 \mu}-a_{1 \mu}\right)+\left(a_{2 \mu}-b_{2 \mu}\right)\right. \\
\left.\quad+\left(a_{3 \mu}-b_{3 \mu}\right)+\left(b_{4 \mu}-a_{4 \mu}\right)\right]+\left[\left(a_{1 v}^{\prime}-b_{1 v}^{\prime}\right)\right. \\
\left.\quad+\left(b_{2 v}^{\prime}-a_{2 v}^{\prime}\right)+\left(b_{3 v}^{\prime}-a_{3 v}^{\prime}\right)+\left(a_{4 v}^{\prime}-b_{4 v}^{\prime}\right)\right]=0 .
\end{gathered}
$$

Therefore from (54) and (55), it is clear that all the terms in (55) are positive and therefore their sum gives zero only when each term is equal to zero. Hence $a_{1 \mu}=b_{1 \mu}, a_{2 \mu}=b_{2 \mu}, a_{3 \mu}=$ $b_{3 \mu}, a_{4 \mu}=b_{4 \mu}$ and $a_{1 v}^{\prime}=b_{1 v}^{\prime}, a_{2 v}^{\prime}=b_{2 v}^{\prime}, a_{3 v}^{\prime}=b_{3 v}^{\prime}, a_{4 v}^{\prime}=b_{4 v}^{\prime}$. Hence $A_{I}=B_{I}$. 
Note 14. The left area score can be calculated to any TrIFN. But $J_{7}$ gives total order on $C_{7}$ which is seen from Theorems 83 and 84.

Definition 85. If $J_{7}\left(A_{I}\right)>J_{7}\left(B_{I}\right)\left(J_{7}\left(A_{I}\right)<J_{7}\left(B_{I}\right)\right)$ then $A_{I}>$ $B_{I}\left(A_{I}<B_{I}\right)$.

Ranking relation defined above is explained in the following example.

Example 86. Let $A_{I}=\langle(0.17,0.23,0.34,0.49),(0.1,0.15,0.4$, $0.6)\rangle$ and $B_{I}=\langle(0.2,0.2,0.3,0.55),(0,0.18,0.47,0.58)\rangle \in C_{7}$. Now $J_{7}\left(A_{I}\right)=-0.0075$ and $J_{7}\left(B_{I}\right)=0.04, J_{7}\left(B_{I}\right)>J_{7}\left(A_{I}\right)$.

Hence $B_{I}>A_{I}$.

The relative importance of the score function $J_{7}$ is explained in the following example.

Example 87. Let $A_{I}=\left\langle\left(a_{1 \mu}, a_{2 \mu}, a_{3 \mu}, a_{4 \mu}\right),\left(a_{1 v}^{\prime}, a_{2 v}^{\prime}, a_{3 v}^{\prime}, a_{4 v}^{\prime}\right)\right\rangle$ and $B_{I}=\left\langle\left(a_{1 \mu}+\epsilon, a_{2 \mu}-\epsilon, a_{3 \mu}-\epsilon, a_{4 \mu}+\epsilon\right),\left(a_{1 \nu}^{\prime}-\epsilon, a_{2 \nu}^{\prime}+\right.\right.$ $\left.\left.\epsilon, a_{3 \nu}^{\prime}+\epsilon, a_{4 v}^{\prime}-\epsilon\right)\right\rangle \in \operatorname{TrIFN}$ and $\epsilon \in[0,1]$.

Now

$$
\begin{aligned}
& J_{1}\left(A_{I}\right)=J_{1}\left(B_{I}\right) \\
& =\frac{\left(a_{1 \mu}+a_{2 \mu}\right)-\left(a_{3 \mu}+a_{4 \mu}\right)+\left(a_{1 \nu}^{\prime}+a_{2 \nu}^{\prime}\right)-\left(a_{3 \nu}^{\prime}+a_{4 \nu}^{\prime}\right)}{8}, \\
& J_{2}\left(A_{I}\right)=J_{2}\left(B_{I}\right) \\
& =\frac{\left(a_{1 \mu}+a_{2 \mu}\right)-\left(a_{3 \mu}+a_{4 \mu}\right)-\left(a_{1 \nu}^{\prime}+a_{2 \nu}^{\prime}\right)+\left(a_{3 \nu}^{\prime}+a_{4 \nu}^{\prime}\right)}{8}, \\
& J_{3}\left(A_{I}\right)=J_{3}\left(B_{I}\right) \\
& =\frac{\left(a_{1 \nu}^{\prime}+a_{2 \nu}^{\prime}+a_{3 \nu}^{\prime}+a_{4 v}^{\prime}\right)-\left(a_{1 \mu}+a_{2 \mu}+a_{3 \mu}+a_{4 \mu}\right)}{8}, \\
& J_{4}\left(A_{I}\right)=J_{4}\left(B_{I}\right) \\
& =\frac{\left(a_{1 \mu}+a_{2 \mu}+a_{3 \mu}+a_{4 \mu}\right)+\left(a_{1 \nu}^{\prime}+a_{2 \nu}^{\prime}+a_{3 \nu}^{\prime}+a_{4 \nu}^{\prime}\right)}{8}, \\
& J_{5}\left(A_{I}\right)=J_{5}\left(B_{I}\right) \\
& =\frac{\left(a_{1 \mu}+a_{3 \mu}\right)-\left(a_{2 \mu}+a_{4 \mu}\right)+\left(a_{1 \nu}^{\prime}+a_{3 \nu}^{\prime}\right)-\left(a_{2 \nu}^{\prime}+a_{4 \nu}^{\prime}\right)}{8}, \\
& J_{6}\left(A_{I}\right)=J_{6}\left(B_{I}\right) \\
& =\frac{\left(a_{2 \mu}+a_{4 \mu}\right)-\left(a_{1 \mu}+a_{3 \mu}\right)+\left(a_{1 \nu}^{\prime}+a_{3 \nu}^{\prime}\right)-\left(a_{2 \nu}^{\prime}+a_{4 \nu}^{\prime}\right)}{8} \text {. }
\end{aligned}
$$

But

$$
\begin{aligned}
& J_{7}\left(\mathrm{~A}_{I}\right) \\
& \quad=\frac{\left(a_{3 \nu}^{\prime}-a_{1 \nu}^{\prime}\right)+\left(a_{2 \nu}^{\prime}-a_{4 v}^{\prime}\right)-\left(a_{3 \mu}-a_{1 \mu}\right)-\left(a_{2 \mu}-a_{4 \mu}\right)}{8}, \\
& J_{7}\left(B_{I}\right) \\
& =\frac{\left(a_{3 \nu}^{\prime}-a_{1 v}^{\prime}\right)+\left(a_{2 v}^{\prime}-a_{4 v}^{\prime}\right)-\left(a_{3 \mu}-a_{1 \mu}\right)-\left(a_{2 \mu}-a_{4 \mu}\right)}{8} \\
& \quad-\epsilon \Longrightarrow \\
& J_{7}\left(A_{I}\right)>J_{7}\left(B_{I}\right) .
\end{aligned}
$$

Hence $A_{I}>B_{I}$.
Example 88. Let $A_{I}=\left\langle\left(a_{1 \mu}, a_{2 \mu}, a_{3 \mu}, a_{4 \mu}\right),\left(a_{1 v}^{\prime}, a_{2 v}^{\prime}, a_{3 v}^{\prime}, a_{4 v}^{\prime}\right)\right\rangle$ and $B_{I}=\left\langle\left(a_{1 \mu}+\epsilon, a_{2 \mu}-\epsilon, a_{3 \mu}-\epsilon, a_{4 \mu}+\epsilon\right),\left(a_{1 v}^{\prime}+\epsilon, a_{2 v}^{\prime}-\epsilon, a_{3 \nu}^{\prime}-\right.\right.$ $\left.\left.\epsilon, a_{4 v}^{\prime}+\epsilon\right)\right\rangle \in \operatorname{TrIFN}$ but not in $C_{7}$, where $\epsilon \in[0,1]$.

Now

$$
\begin{aligned}
& J_{1}\left(A_{I}\right)=J_{1}\left(B_{I}\right) \\
& =\frac{\left(a_{1 \mu}+a_{2 \mu}\right)-\left(a_{3 \mu}+a_{4 \mu}\right)+\left(a_{1 \nu}^{\prime}+a_{2 \nu}^{\prime}\right)-\left(a_{3 \nu}^{\prime}+a_{4 v}^{\prime}\right)}{8}, \\
& J_{2}\left(A_{I}\right)=J_{2}\left(B_{I}\right) \\
& =\frac{\left(a_{1 \mu}+a_{2 \mu}\right)-\left(a_{3 \mu}+a_{4 \mu}\right)-\left(a_{1 \nu}^{\prime}+a_{2 \nu}^{\prime}\right)+\left(a_{3 \nu}^{\prime}+a_{4 \nu}^{\prime}\right)}{8}, \\
& J_{3}\left(A_{I}\right)=J_{3}\left(B_{I}\right) \\
& =\frac{\left(a_{1 \nu}^{\prime}+a_{2 \nu}^{\prime}+a_{3 \nu}^{\prime}+a_{4 \nu}^{\prime}\right)-\left(a_{1 \mu}+a_{2 \mu}+a_{3 \mu}+a_{4 \mu}\right)}{8}, \\
& J_{4}\left(A_{I}\right)=J_{4}\left(B_{I}\right) \\
& =\frac{\left(a_{1 \mu}+a_{2 \mu}+a_{3 \mu}+a_{4 \mu}\right)+\left(a_{1 \nu}^{\prime}+a_{2 \nu}^{\prime}+a_{3 \nu}^{\prime}+a_{4 \nu}^{\prime}\right)}{8}, \\
& J_{5}\left(A_{I}\right)=J_{5}\left(B_{I}\right) \\
& =\frac{\left(a_{1 \mu}+a_{3 \mu}\right)-\left(a_{2 \mu}+a_{4 \mu}\right)+\left(a_{1 \nu}^{\prime}+a_{3 \nu}^{\prime}\right)-\left(a_{2 \nu}^{\prime}+a_{4 \nu}^{\prime}\right)}{8}, \\
& J_{6}\left(A_{I}\right)=J_{6}\left(B_{I}\right) \\
& =\frac{\left(a_{2 \mu}+a_{4 \mu}\right)-\left(a_{1 \mu}+a_{3 \mu}\right)+\left(a_{1 \nu}^{\prime}+a_{3 \nu}^{\prime}\right)-\left(a_{2 \nu}^{\prime}+a_{4 \nu}^{\prime}\right)}{8}, \\
& J_{7}\left(A_{I}\right)=J_{7}\left(B_{I}\right) \\
& =\frac{\left(a_{3 \nu}^{\prime}-a_{1 \nu}^{\prime}\right)+\left(a_{2 v}^{\prime}-a_{4 \nu}^{\prime}\right)-\left(a_{3 \mu}-a_{1 \mu}\right)-\left(a_{2 \mu}-a_{4 \mu}\right)}{8} .
\end{aligned}
$$

But $A_{I} \neq B_{I}$.

Pictorial representation of this example is given in Figure 9.

Example 87 shows that all the above defined scores are not enough to cover the entire class of TrIFNs. Hence the new score function is introduced in the next subsection in order to give total ordering on the entire class of TrIFNs.

3.8. Right Area Score of a Trapezoidal Intuitionistic Fuzzy Number. In this subsection, a new subclass of TrIFNs is introduced and the right area score function on this class of trapezoidal intuitionistic fuzzy numbers is defined and some of its properties are studied using illustrative examples.

Definition 89. Let $A_{I}$ and $B_{I}$ be two TrIFNs.

A special subclass $C_{8}$ of the set of TrIFNs consist of TrIFNs for which every pair of $A_{I}$ and $B_{I}$ are with $a_{1 \mu} \leq b_{1 \mu}, a_{2 \mu} \geq$ $b_{2 \mu}, a_{3 \mu} \geq b_{3 \mu}, a_{4 \mu} \leq b_{4 \mu}$ and $a_{1 \nu}^{\prime} \leq b_{1 \nu}^{\prime}, a_{2 \nu}^{\prime} \geq b_{2 \nu}^{\prime}, a_{3 \nu}^{\prime} \geq$ $b_{3 v}^{\prime}, a_{4 v}^{\prime} \leq b_{4 v}^{\prime}$. The definite relation on $C_{8}$ is denoted as $\gg$ and defined as follows: if $A_{I}, B_{I} \in C_{8} \subset$ TrIFN such that $A_{I} \gg B_{I}$ then $a_{1 \mu} \leq b_{1 \mu}, a_{2 \mu} \geq b_{2 \mu}, a_{3 \mu} \geq b_{3 \mu}, a_{4 \mu} \leq b_{4 \mu}$ and 


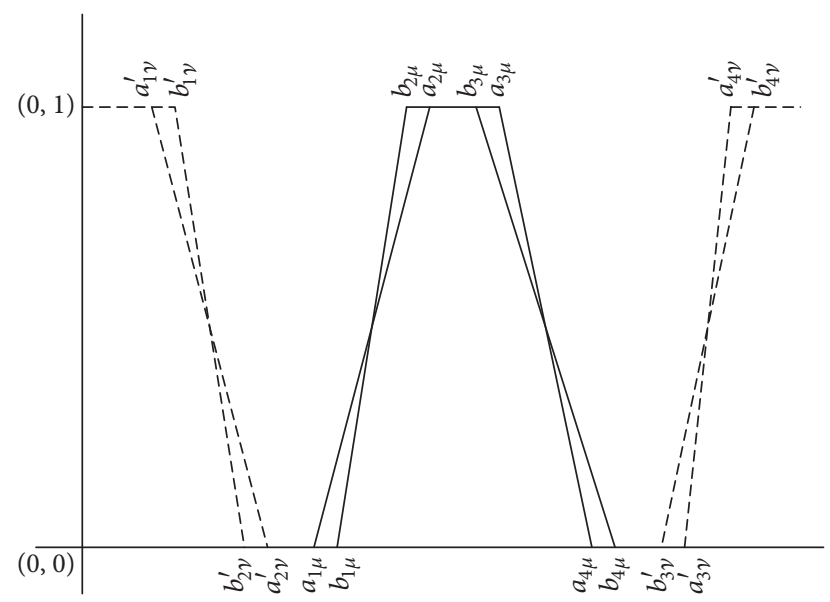

FIGURE 9: Pictorial representation of Example 88.

$a_{1 v}^{\prime} \leq b_{1 v}^{\prime}, a_{2 v}^{\prime} \geq b_{2 v}^{\prime}, a_{3 v}^{\prime} \geq b_{3 v}^{\prime}, a_{4 v}^{\prime} \leq b_{4 v}^{\prime}$. If $A_{I} \gg B_{I}$ then one of the above inequalities becomes strict inequality.

Note 15. By Definition 89, we note that any pair of members of $C_{8}$ are related under $\gg$.

The score function which measures the right area is defined as follows.

Definition 90. Let $A_{I}$ be a trapezoidal intuitionistic fuzzy number. Then the right area score of $A_{I}$ is defined by

$$
\begin{aligned}
& J_{8}\left(A_{I}\right) \\
& =\frac{\left(a_{1 \mu}-a_{3 \mu}\right)+\left(a_{4 \mu}-a_{2 \mu}\right)+\left(a_{1 \nu}^{\prime}-a_{3} \nu^{\prime}\right)+\left(a_{4 \nu}^{\prime}-a_{2 \nu}^{\prime}\right)}{8} .
\end{aligned}
$$

The proofs of the following propositions are immediate from Definition 90 and hence they are omitted.

Proposition 91. For any real number $r \in[0,1], J_{8}(r)=0$.

Proposition 92. If $A_{I}=\left(a_{1 \mu}, a_{2 \mu}, a_{3 \mu}, a_{4 \mu}\right)$ is a trapezoidal fuzzy number, then $J_{8}\left(A_{I}\right)=\left(\left(a_{1 \mu}-a_{3 \mu}\right)+\left(a_{4 \mu}-a_{2 \mu}\right)\right) / 4$.

Proposition 93. Let $A_{I}=\left\langle\left(a_{1 \mu}, a_{2 \mu}, a_{3 \mu}\right),\left(a_{1 v}^{\prime}, a_{2 v}^{\prime}, a_{3 v}^{\prime}\right)\right\rangle$ be a triangular intuitionistic fuzzy number. Then $J_{8}\left(A_{I}\right)=\left(\left(a_{1 \mu}+\right.\right.$ $\left.\left.a_{3 \mu}-2 a_{2 \mu}\right)+\left(a_{1 \nu}^{\prime}+a_{3 \nu}^{\prime}-2 a_{2 v}^{\prime}\right)\right) / 8$.

Proposition 94. Let $A_{I}=\left(\left[a_{1 \mu}, a_{2 \mu}\right],\left[a_{1 v}^{\prime}, a_{2 v}^{\prime}\right]\right)$ be an interval valued intuitionistic fuzzy number. Then $J_{8}\left(A_{I}\right)=0$.

Note 16. The right area score can be calculated to any TrIFN. But $J_{8}$ gives total order on $C_{8}$ which is proved in the following theorems.

Theorem 95. Let $A_{I}, B_{I} \in C_{8}$. If $A_{I} \gg B_{I}$ then $J_{8}\left(A_{I}\right)<$ $J_{8}\left(B_{I}\right)$.

Proof. Let us assume that $A_{I} \gg B_{I}$. We claim that $J_{8}\left(B_{I}\right)-$ $J_{8}\left(A_{I}\right)>0$.
By Definition 89, we have

$$
\begin{aligned}
& a_{1 \mu} \leq b_{1 \mu}, \\
& a_{2 \mu} \geq b_{2 \mu}, \\
& a_{3 \mu} \geq b_{3 \mu}, \\
& a_{4 \mu} \leq b_{4 \mu}, \\
& a_{1 v}^{\prime} \leq b_{1 v}^{\prime}, \\
& a_{2 v}^{\prime} \geq b_{2 v}^{\prime}, \\
& a_{3 v}^{\prime} \geq b_{3 v}^{\prime}, \\
& a_{4 v}^{\prime} \leq b_{4 v}^{\prime} .
\end{aligned}
$$

Now,

$$
\begin{gathered}
8\left(J_{8}\left(B_{I}\right)-J_{8}\left(A_{I}\right)\right)=\left[\left(b_{1 \mu}-a_{1 \mu}\right)+\left(a_{2 \mu}-b_{2 \mu}\right)\right. \\
\left.+\left(a_{3 \mu}-b_{3 \mu}\right)+\left(b_{4 \mu}-a_{4 \mu}\right)\right]+\left[\left(b_{1 \nu}^{\prime}-a_{1 \nu}^{\prime}\right)\right. \\
\left.+\left(a_{2 \nu}^{\prime}-b_{2 \nu}^{\prime}\right)+\left(a_{3 \nu}^{\prime}-b_{3 \nu}^{\prime}\right)+\left(b_{4 \nu}^{\prime}-a_{4 \nu}^{\prime}\right)\right] .
\end{gathered}
$$

From (60) it is very easy to see that all the terms in (61) are positive. Therefore their sum is also positive. From Definition 89 , we know that at least one of the above inequalities in $(60)$ becomes strict inequality and hence we get $J_{8}\left(B_{I}\right)-$ $J_{8}\left(A_{I}\right)>0$.

Theorem 96. Let $A_{I}=\left\langle\left(a_{1 \mu}, a_{2 \mu}, a_{3 \mu}, a_{4 \mu}\right),\left(a_{1 v}^{\prime}, a_{2 v}^{\prime}, a_{3 v}^{\prime}, a_{4 v}^{\prime}\right)\right\rangle$ and $B_{I}=\left\langle\left(b_{1 \mu}, b_{2 \mu}, b_{3 \mu}, b_{4 \mu}\right),\left(b_{1 v}^{\prime}, b_{2 v}^{\prime}, b_{3 v}^{\prime}, b_{4 v}^{\prime}\right)\right\rangle \in C_{8}$ such that $J_{8}\left(A_{I}\right)=J_{8}\left(B_{I}\right)$; then $A_{I}=B_{I}$.

Proof. Let us assume $J_{8}\left(A_{I}\right)=J_{8}\left(B_{I}\right)$. We claim that $A_{I}=B_{I}$. By Definition 89, without loss of generality, we have

$$
\begin{aligned}
& a_{1 \mu} \leq b_{1 \mu}, \\
& a_{2 \mu} \geq b_{2 \mu}, \\
& a_{3 \mu} \geq b_{3 \mu}, \\
& a_{4 \mu} \leq b_{4 \mu}, \\
& a_{1 v}^{\prime} \leq b_{1 v}^{\prime}, \\
& a_{2 v}^{\prime} \geq b_{2 v}^{\prime}, \\
& a_{3 v}^{\prime} \geq b_{3 v}^{\prime}, \\
& a_{4 v}^{\prime} \leq b_{4 v}^{\prime} .
\end{aligned}
$$

Now,

$$
\begin{gathered}
8\left(J_{1}\left(B_{I}\right)-J_{1}\left(A_{I}\right)\right)=\left[\left(b_{1 \mu}-a_{1 \mu}\right)+\left(a_{2 \mu}-b_{2 \mu}\right)\right. \\
\left.+\left(a_{3 \mu}-b_{3 \mu}\right)+\left(b_{4 \mu}-a_{4 \mu}\right)\right]+\left[\left(b_{1 \nu}^{\prime}-a_{1 \nu}^{\prime}\right)\right. \\
\left.+\left(a_{2 \nu}^{\prime}-b_{2 \nu}^{\prime}\right)+\left(a_{3 \nu}^{\prime}-b_{3 \nu}^{\prime}\right)+\left(b_{4 \nu}^{\prime}-a_{4 \nu}^{\prime}\right)\right]=0 .
\end{gathered}
$$


Therefore from (62) and (63), it is clear that all the terms in (63) are positive and therefore their sum gives zero only when each term is equal to zero. Hence $a_{1 \mu}=b_{1 \mu}, a_{2 \mu}=b_{2 \mu}, a_{3 \mu}=$ $b_{3 \mu}, a_{4 \mu}=b_{4 \mu}$ and $a_{1 v}^{\prime}=b_{1 v}^{\prime}, a_{2 v}^{\prime}=b_{2 v}^{\prime}, a_{3 v}^{\prime}=b_{3 v}^{\prime}, a_{4 v}^{\prime}=b_{4 v}^{\prime}$, hence the proof.

Definition 97. If $J_{8}\left(A_{I}\right)>J_{8}\left(B_{I}\right)\left(J_{8}\left(A_{I}\right)<J_{8}\left(B_{I}\right)\right)$ then $A_{I}>$ $B_{I}\left(A_{I}<B_{I}\right)$.

Ranking procedure introduced in Definition 97 is demonstrated in Example 98.

Example 98. Let $A_{I}=\langle(0.1,0.2,0.3,0.45),(0,0.15,0.35$, $0.5)\rangle$ and $B_{I}=\langle(0.15,0.15,0.2,0.45),(0.1,0.1,0.3,0.45)\rangle \in$ $C_{8}$. Now $J_{8}\left(A_{I}\right)=0.00625$ and $J_{8}\left(B_{I}\right)=0.05, J_{8}\left(B_{I}\right)>$ $J_{8}\left(A_{I}\right)$.

Hence $B_{I}>A_{I}$.

The relative importance of the score function $J_{8}$ in ranking arbitrary TrIFNs is explained mathematically in the following example.

Example 99. Let $A_{I}=\left\langle\left(a_{1 \mu}, a_{2 \mu}, a_{3 \mu}, a_{4 \mu}\right),\left(a_{1 v}^{\prime}, a_{2 v}^{\prime}, a_{3 v}^{\prime}, a_{4 v}^{\prime}\right)\right\rangle$ and $B_{I}=\left\langle\left(a_{1 \mu}+\epsilon, a_{2 \mu}-\epsilon, a_{3 \mu}-\epsilon, a_{4 \mu}+\epsilon\right),\left(a_{1 \nu}^{\prime}+\epsilon, a_{2 v}^{\prime}-\epsilon, a_{3 \nu}^{\prime}-\right.\right.$ $\left.\left.\epsilon, a_{4 v}^{\prime}+\epsilon\right)\right\rangle \in \operatorname{TrIFN}$ but not in $C_{7}$, where $\epsilon \in[0,1]$.

Now

$$
\begin{aligned}
& J_{1}\left(A_{I}\right)=J_{1}\left(B_{I}\right) \\
& =\frac{\left(a_{1 \mu}+a_{2 \mu}\right)-\left(a_{3 \mu}+a_{4 \mu}\right)+\left(a_{1 \nu}^{\prime}+a_{2 \nu}^{\prime}\right)-\left(a_{3 \nu}^{\prime}+a_{4 \nu}^{\prime}\right)}{8}, \\
& J_{2}\left(A_{I}\right)=J_{2}\left(B_{I}\right) \\
& =\frac{\left(a_{1 \mu}+a_{2 \mu}\right)-\left(a_{3 \mu}+a_{4 \mu}\right)-\left(a_{1 \nu}^{\prime}+a_{2 \nu}^{\prime}\right)+\left(a_{3 \nu}^{\prime}+a_{4 \nu}^{\prime}\right)}{8}, \\
& J_{3}\left(A_{I}\right)=J_{3}\left(B_{I}\right) \\
& =\frac{\left(a_{1 \nu}^{\prime}+a_{2 \nu}^{\prime}+a_{3 \nu}^{\prime}+a_{4 \nu}^{\prime}\right)-\left(a_{1 \mu}+a_{2 \mu}+a_{3 \mu}+a_{4 \mu}\right)}{8}, \\
& J_{4}\left(A_{I}\right)=J_{4}\left(B_{I}\right) \\
& =\frac{\left(a_{1 \mu}+a_{2 \mu}+a_{3 \mu}+a_{4 \mu}\right)+\left(a_{1 \nu}^{\prime}+a_{2 \nu}^{\prime}+a_{3 \nu}^{\prime}+a_{4 \nu}^{\prime}\right)}{8}, \\
& J_{5}\left(A_{I}\right)=J_{5}\left(B_{I}\right) \\
& =\frac{\left(a_{1 \mu}+a_{3 \mu}\right)-\left(a_{2 \mu}+a_{4 \mu}\right)+\left(a_{1 \nu}^{\prime}+a_{3 \nu}^{\prime}\right)-\left(a_{2 \nu}^{\prime}+a_{4 \nu}^{\prime}\right)}{8}, \\
& J_{6}\left(A_{I}\right)=J_{6}\left(B_{I}\right) \\
& =\frac{\left(a_{2 \mu}+a_{4 \mu}\right)-\left(a_{1 \mu}+a_{3 \mu}\right)+\left(a_{1 \nu}^{\prime}+a_{3 \nu}^{\prime}\right)-\left(a_{2 \nu}^{\prime}+a_{4 \nu}^{\prime}\right)}{8}, \\
& J_{7}\left(A_{I}\right)=J_{7}\left(B_{I}\right) \\
& =\frac{\left(a_{3 \nu}^{\prime}-a_{1 \nu}^{\prime}\right)+\left(a_{2 \nu}^{\prime}-a_{4 \nu}^{\prime}\right)-\left(a_{3 \mu}-a_{1 \mu}\right)-\left(a_{2 \mu}-a_{4 \mu}\right)}{8} \text {. }
\end{aligned}
$$

But

$$
\begin{aligned}
& J_{8}\left(A_{I}\right) \\
& =\frac{\left(a_{1 \mu}-a_{3 \mu}\right)+\left(a_{4 \mu}-a_{2 \mu}\right)+\left(a_{1 v}^{\prime}-a_{3 \nu}^{\prime}\right)+\left(a_{4 v}^{\prime}-a_{2 v}^{\prime}\right)}{8}, \\
& J_{8}\left(B_{I}\right) \\
& =\frac{\left(a_{1 \mu}-a_{3 \mu}\right)+\left(a_{4 \mu}-a_{2 \mu}\right)+\left(a_{1 v}^{\prime}-a_{3 v}^{\prime}\right)+\left(a_{4 v}^{\prime}-a_{2 v}^{\prime}\right)}{8} \\
& \quad-\epsilon \Longrightarrow \\
& J_{8}\left(A_{I}\right)>J_{8}\left(B_{I}\right) .
\end{aligned}
$$

Hence $A_{I}>B_{I}$.

\section{A New Ranking Principle for Ordering Trapezoidal Intuitionistic Fuzzy Numbers}

In this section a new ranking principle on trapezoidal intuitionistic fuzzy numbers is defined by using finite number of score functions defined in Sections 3.1, 3.2, 3.3, 3.4, 3.5, 3.6, 3.7, and 3.8 and we proved that the proposed ranking is a total order on the class of TrIFNs.

Definition 100. Let $A_{I}, B_{I} \in \operatorname{TrIFN}$. An order relation < on the set of TrIFNs is defined by the following:

If $J_{1}\left(A_{I}\right)<J_{1}\left(B_{I}\right)$ then $A_{I}$ is smaller than $B_{I}$, denoted by $A_{I}<B_{I}$.

If $J_{1}\left(A_{I}\right)=J_{1}\left(B_{I}\right)$ thenif $J_{2}\left(A_{I}\right)<J_{2}\left(B_{I}\right)$ then $A_{I}$ is smaller than $B_{I}$, denoted by $A_{I}<B_{I}$.

If $J_{1}\left(A_{I}\right)=J_{1}\left(B_{I}\right)$ and $J_{2}\left(A_{I}\right)=J_{2}\left(B_{I}\right)$ then if $J_{3}\left(A_{I}\right)<J_{3}\left(B_{I}\right)$ then $A_{I}$ is smaller than $B_{I}$, denoted by $A_{I}<B_{I}$.

If $J_{1}\left(A_{I}\right)=J_{1}\left(B_{I}\right), J_{2}\left(A_{I}\right)=J_{2}\left(B_{I}\right)$, and $J_{3}\left(A_{I}\right)=$ $J_{3}\left(B_{I}\right)$ then if $J_{4}\left(A_{I}\right)<J_{4}\left(B_{I}\right)$ then $A_{I}$ is smaller than $B_{I}$, denoted by $A_{I}<B_{I}$.

If $J_{1}\left(A_{I}\right)=J_{1}\left(B_{I}\right), J_{2}\left(A_{I}\right)=J_{2}\left(B_{I}\right), J_{3}\left(A_{I}\right)=J_{3}\left(B_{I}\right)$, and $J_{4}\left(A_{I}\right)=J_{4}\left(B_{I}\right)$ then if $J_{5}\left(A_{I}\right)<J_{5}\left(B_{I}\right)$ then $A_{I}$ is smaller than $B_{I}$, denoted by $A_{I}<B_{I}$.

If $J_{1}\left(A_{I}\right)=J_{1}\left(B_{I}\right), J_{2}\left(A_{I}\right)=J_{2}\left(B_{I}\right), J_{3}\left(A_{I}\right)=J_{3}\left(B_{I}\right)$, $J_{4}\left(A_{I}\right)=J_{4}\left(B_{I}\right)$, and $J_{5}\left(A_{I}\right)=J_{5}\left(B_{I}\right)$ then if $J_{6}\left(A_{I}\right)<$ $J_{6}\left(B_{I}\right)$ then $A_{I}$ is smaller than $B_{I}$, denoted by $A_{I}<B_{I}$.

If $J_{1}\left(A_{I}\right)=J_{1}\left(B_{I}\right), J_{2}\left(A_{I}\right)=J_{2}\left(B_{I}\right), J_{3}\left(A_{I}\right)=J_{3}\left(B_{I}\right)$, $J_{4}\left(A_{I}\right)=J_{4}\left(B_{I}\right), J_{5}\left(A_{I}\right)=J_{5}\left(B_{I}\right)$, and $J_{6}\left(A_{I}\right)=J_{6}\left(B_{I}\right)$ then if $J_{7}\left(A_{I}\right)<J_{7}\left(B_{I}\right)$ then $A_{I}$ is smaller than $B_{I}$, denoted by $A_{I}<B_{I}$.

If $J_{1}\left(A_{I}\right)=J_{1}\left(B_{I}\right), J_{2}\left(A_{I}\right)=J_{2}\left(B_{I}\right), J_{3}\left(A_{I}\right)=J_{3}\left(B_{I}\right)$, $J_{4}\left(A_{I}\right)=J_{4}\left(B_{I}\right), J_{5}\left(A_{I}\right)=J_{5}\left(B_{I}\right), J_{6}\left(A_{I}\right)=J_{6}\left(B_{I}\right)$, and $J_{7}\left(A_{I}\right)=J_{7}\left(B_{I}\right)$ then if $J_{8}\left(A_{I}\right)<J_{8}\left(B_{I}\right)$ then $A_{I}$ is smaller than $B_{I}$, denoted by $A_{I}<B_{I}$.

If $J_{1}\left(A_{I}\right)=J_{1}\left(B_{I}\right), J_{2}\left(A_{I}\right)=J_{2}\left(B_{I}\right), J_{3}\left(A_{I}\right)=J_{3}\left(B_{I}\right)$, $J_{4}\left(A_{I}\right)=J_{4}\left(B_{I}\right), J_{5}\left(A_{I}\right)=J_{5}\left(B_{I}\right), J_{6}\left(A_{I}\right)=J_{6}\left(B_{I}\right)$, $J_{7}\left(A_{I}\right)=J_{7}\left(B_{I}\right)$, and $J_{8}\left(A_{I}\right)=J_{8}\left(B_{I}\right)$ then $A_{I}=B_{I}$. 
The following theorem is proved to show the validity of Definition 100.

Theorem 101. Let $A_{I}, B_{I} \in \operatorname{TrIFN}$. If $J_{1}\left(A_{I}\right)=J_{1}\left(B_{I}\right)$, $J_{2}\left(A_{I}\right)=J_{2}\left(B_{I}\right), J_{3}\left(A_{I}\right)=J_{3}\left(B_{I}\right), J_{4}\left(A_{I}\right)=J_{4}\left(B_{I}\right), J_{5}\left(A_{I}\right)=$ $J_{5}\left(B_{I}\right), J_{6}\left(A_{I}\right)=J_{6}\left(B_{I}\right), J_{7}\left(A_{I}\right)=J_{7}\left(B_{I}\right)$, and $J_{8}\left(A_{I}\right)=J_{8}\left(B_{I}\right)$ then $A_{I}=B_{I}$. That is, $a_{1 \mu}=b_{1 \mu}, a_{2 \mu}=b_{2 \mu}, a_{3 \mu}=b_{3 \mu}, a_{4 \mu}=$ $b_{4 \mu}$, and $a_{1 v}^{\prime}=b_{1 v}^{\prime}, a_{2 v}^{\prime}=b_{2 v}^{\prime}, a_{3 v}^{\prime}=b_{3 v}^{\prime}, a_{4 v}^{\prime}=b_{4 v}^{\prime}$.

Proof. Let $A_{I}, B_{I} \in \operatorname{TrIFN}$. Let us assume $J_{1}\left(A_{I}\right)=J_{1}\left(B_{I}\right)$, $J_{2}\left(A_{I}\right)=J_{2}\left(B_{I}\right), J_{3}\left(A_{I}\right)=J_{3}\left(B_{I}\right), J_{4}\left(A_{I}\right)=J_{4}\left(B_{I}\right), J_{5}\left(A_{I}\right)=$ $J_{5}\left(B_{I}\right), J_{6}\left(A_{I}\right)=J_{6}\left(B_{I}\right), J_{7}\left(A_{I}\right)=J_{7}\left(B_{I}\right)$, and $J_{8}\left(A_{I}\right)=J_{8}\left(B_{I}\right)$.

Claim $1\left(A_{I}=B_{I}\right)$.

$$
\begin{aligned}
& -J_{1}\left(A_{I}\right)+J_{4}\left(A_{I}\right)=-J_{1}\left(B_{I}\right)+J_{4}\left(B_{I}\right) \Longrightarrow \\
& \left(a_{3 \mu}+a_{4 \mu}\right)+\left(a_{3 \nu}^{\prime}+a_{4 \nu}^{\prime}\right)=\left(b_{3 \mu}+b_{4 \mu}\right)+\left(b_{3 \nu}^{\prime}+b_{4 v}^{\prime}\right) \\
& J_{4}\left(A_{I}\right)+J_{1}\left(A_{I}\right)=J_{4}\left(B_{I}\right)+J_{1}\left(A_{I}\right) \Longrightarrow \\
& \left(a_{1 \mu}+a_{2 \mu}\right)+\left(a_{1 \nu}^{\prime}+a_{2 \nu}^{\prime}\right)=\left(b_{1 \mu}+b_{2 \mu}\right)+\left(b_{1 \nu}^{\prime}+b_{2 v}^{\prime}\right) \\
& J_{2}\left(A_{I}\right)+J_{4}\left(A_{I}\right)=J_{2}\left(B_{I}\right)+J_{4}\left(B_{I}\right) \Longrightarrow \\
& \left(a_{1 \mu}+a_{2 \mu}\right)+\left(a_{3 \nu}^{\prime}+a_{4 \nu}^{\prime}\right)=\left(b_{1 \mu}+b_{2 \mu}\right)+\left(b_{3 \nu}^{\prime}+b_{4 v}^{\prime}\right) \\
& -J_{3}\left(A_{I}\right)+J_{4}\left(A_{I}\right)=-J_{3}\left(B_{I}\right)+J_{4}\left(B_{I}\right) \Longrightarrow \\
& \left(a_{1 \mu}+a_{2 \mu}\right)+\left(a_{3 \nu}+a_{4 \nu}\right)=\left(b_{1 \mu}+b_{2 \mu}\right)+\left(b_{3 \nu}+b_{4 v}\right) \\
& (68)-(66) \Longrightarrow\left(a_{1 \mu}+a_{2 \mu}\right)-\left(a_{3 \nu}+a_{4 \nu}\right) \\
& =\left(b_{1 \mu}+b_{2 \mu}\right)-\left(b_{3 \nu}+b_{4 \nu}\right) \\
& (69)+(70) \Longrightarrow\left(a_{1 \mu}+a_{2 \mu}\right)=\left(b_{1 \mu}+b_{2 \mu}\right) \\
& (69)-(70) \Longrightarrow\left(a_{3 \mu}+a_{4 \mu}\right)=\left(b_{3 \mu}+b_{4 \mu}\right) .
\end{aligned}
$$

From (71), (66), and (67), we get

$$
\begin{aligned}
& \left(a_{1 v}^{\prime}+a_{2 v}^{\prime}\right)=\left(b_{1 v}^{\prime}+b_{2 v}^{\prime}\right), \\
& \left(a_{3 v}^{\prime}+a_{4 v}^{\prime}\right)=\left(b_{3 v}^{\prime}+b_{4 v}^{\prime}\right), \\
& J_{4}\left(A_{I}\right)-J_{8}\left(A_{I}\right)=J_{4}\left(B_{I}\right)-J_{8}\left(B_{I}\right) \Longrightarrow \\
& \left(a_{2 \mu}+a_{3 \mu}\right)+\left(a_{2 v}^{\prime}+a_{3 v}^{\prime}\right)=\left(b_{2 \mu}+b_{3 \mu}\right)+\left(b_{2 v}^{\prime}+b_{3 v}^{\prime}\right), \\
& J_{4}\left(A_{I}\right)-J_{7}\left(A_{I}\right)=J_{4}\left(B_{I}\right)-J_{7}\left(B_{I}\right) \Longrightarrow \\
& \left(a_{2 \mu}+a_{3 \mu}\right)+\left(a_{1 v}^{\prime}+a_{4 v}^{\prime}\right)=\left(b_{2 \mu}+b_{3 \mu}\right)+\left(b_{1 v}^{\prime}+b_{4 v}^{\prime}\right), \\
& J_{4}\left(A_{I}\right)-J_{6}\left(A_{I}\right)=J_{4}\left(B_{I}\right)-J_{6}\left(B_{I}\right) \Longrightarrow \\
& \left(a_{1 \mu}+a_{3 \mu}\right)+\left(a_{2 v}^{\prime}+a_{4 v}^{\prime}\right)=\left(b_{1 \mu}+b_{3 \mu}\right)+\left(b_{2 v}^{\prime}+b_{4 v}^{\prime}\right), \\
& J_{4}\left(A_{I}\right)-J_{5}\left(A_{I}\right)=J_{4}\left(B_{I}\right)-J_{5}\left(B_{I}\right) \Longrightarrow \\
& \left(a_{1 \mu}+a_{3 \mu}\right)+\left(a_{1 v}^{\prime}+a_{3 v}^{\prime}\right)=\left(b_{1 \mu}+b_{3 \mu}\right)+\left(b_{1 v}^{\prime}+b_{3 v}^{\prime}\right),
\end{aligned}
$$

$$
\begin{aligned}
& (75)-(76) \Longrightarrow\left(a_{2 v}^{\prime}-a_{1 v}^{\prime}\right)+\left(a_{3 v}^{\prime}-a_{4 v}^{\prime}\right) \\
& =\left(b_{2 v}^{\prime}-b_{1 v}^{\prime}\right)+\left(b_{3 v}^{\prime}-b_{4 v}^{\prime}\right) \text {, } \\
& (77)-(78) \Longrightarrow\left(a_{2 v}^{\prime}-a_{1 v}^{\prime}\right)+\left(a_{4 v}^{\prime}-a_{3 v}^{\prime}\right) \\
& =\left(b_{2 v}^{\prime}-b_{1 v}^{\prime}\right)+\left(b_{4 v}^{\prime}-b_{3 v}^{\prime}\right), \\
& (79)+(80) \Longrightarrow\left(a_{2 v}^{\prime}-a_{1 v}^{\prime}\right)=\left(b_{2 v}^{\prime}-b_{1 v}^{\prime}\right) \text {, } \\
& (80)-(79) \Longrightarrow\left(a_{4 v}^{\prime}-a_{3 \nu}^{\prime}\right)=\left(b_{4 v}^{\prime}-b_{3 v}^{\prime}\right) \text {, } \\
& J_{2}\left(A_{I}\right)+J_{8}\left(A_{I}\right)=J_{2}\left(B_{I}\right)+J_{8}\left(B_{I}\right) \Longrightarrow \\
& \left(a_{1 \mu}-a_{3 \mu}\right)+\left(a_{4 \nu}^{\prime}-a_{2 \nu}^{\prime}\right)=\left(b_{1 \mu}-b_{3 \mu}\right)+\left(b_{4 \nu}^{\prime}-b_{2 \nu}^{\prime}\right), \\
& -\left(J_{6}\left(A_{I}\right)+J_{7}\left(A_{I}\right)\right)=-\left(J_{6}\left(B_{I}\right)+J_{7}\left(B_{I}\right)\right) \Longrightarrow \\
& \left(a_{3 \mu}-a_{4 \mu}\right)+\left(a_{4 v}^{\prime}-a_{3 \nu}^{\prime}\right)=\left(b_{3 \mu}-b_{4 \mu}\right)+\left(a_{4 \nu}^{\prime}-b_{3 \nu}^{\prime}\right) \text {. }
\end{aligned}
$$

From (73) and (74) and (81) and (82), we get

$$
\begin{aligned}
& a_{1 v}^{\prime}=b_{1 v}^{\prime}, \\
& a_{2 v}^{\prime}=b_{2 v}^{\prime}, \\
& a_{3 v}^{\prime}=b_{3 \nu}^{\prime}, \\
& a_{4 v}^{\prime}=b_{4 v}^{\prime} .
\end{aligned}
$$

From (83), (84), and (85), we get

$$
\begin{aligned}
& \left(a_{1 \mu}-a_{3 \mu}\right)=\left(b_{1 \mu}-b_{3 \mu}\right), \\
& \left(a_{3 \mu}-a_{4 \mu}\right)=\left(b_{3 \mu}-b_{4 \mu}\right) .
\end{aligned}
$$

$(72)+(87) \Rightarrow a_{3 \mu}=b_{3 \mu}$ and (72) $-(87) \Rightarrow a_{4}=b_{4}$. From (86) $a_{3 \mu}=b_{3 \mu}$ implies $a_{1 \mu}=b_{1 \mu}$. Put $a_{1 \mu}=b_{1 \mu}$ in (71) and we get $a_{2 \mu}=b_{2 \mu}$. Therefore $a_{1 \mu}=b_{1 \mu}, a_{2 \mu}=b_{2 \mu}, a_{3 \mu}=b_{3 \mu}, a_{4 \mu}=$ $b_{4 \mu}$ and $a_{1 \nu}^{\prime}=b_{1 \nu}^{\prime}, a_{2 \nu}^{\prime}=b_{2 v}^{\prime}, a_{3 \nu}^{\prime}=b_{3 \nu}^{\prime}, a_{4 \nu}^{\prime}=b_{4 v}^{\prime}$, hence the proof.

Theorem 101 shows that the proposed ordering on TrIFNs satisfies Law of trichotomy and therefore the proposed ranking principle on TrIFNs gives total order on the class of TrIFNs.

Remark 102. The validation of the proposed ranking principle on the subclasses of TrIFNs is listed as follows:

(1) Accuracy score $\left(J_{4}\right)$ function is sufficient to rank the set of real numbers $\Re$ totally, since $J_{1}, J_{2}$, and $J_{3}$ scores become zero for any real number in $[0,1]$.

(2) $J_{1}, J_{4}, J_{5}$, and $J_{8}$ give the total order on the class of trapezoidal fuzzy number.

(3) $J_{1}, J_{2}, J_{3}$, and $J_{4}$ together give the total order on the class of interval valued intuitionistic fuzzy number.

(4) $J_{1}, J_{2}, J_{3}, J_{4}, J_{7}$, and $J_{8}$ provide a complete ranking on the class of triangular intuitionistic fuzzy numbers. 


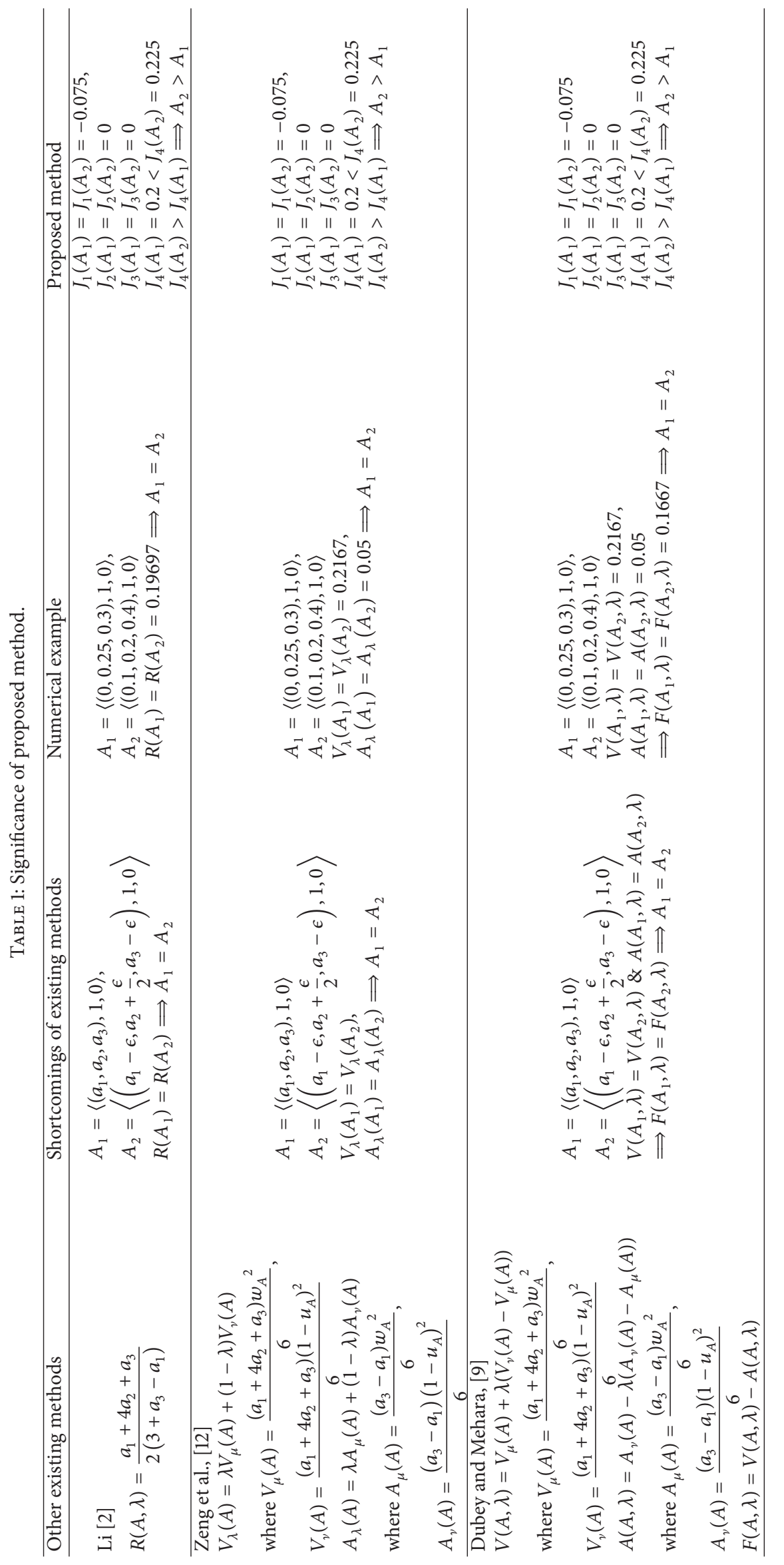




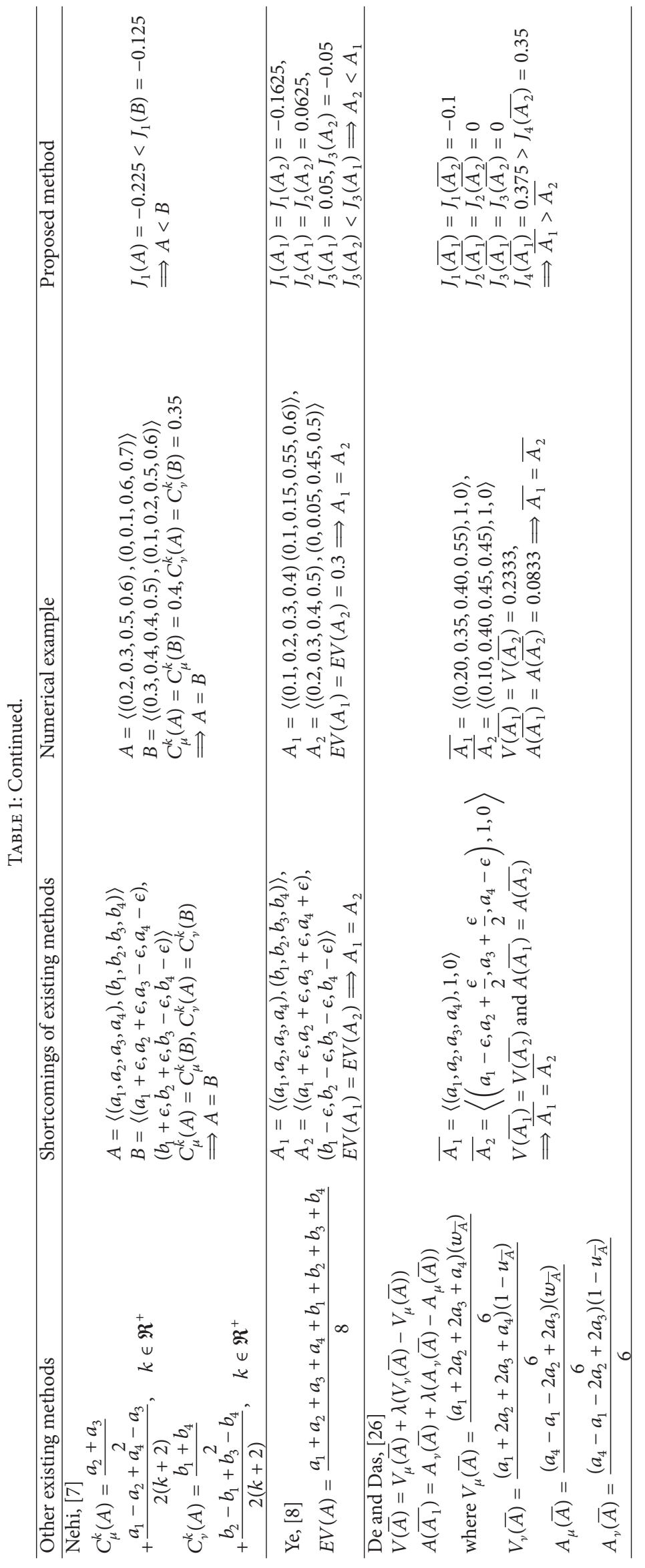




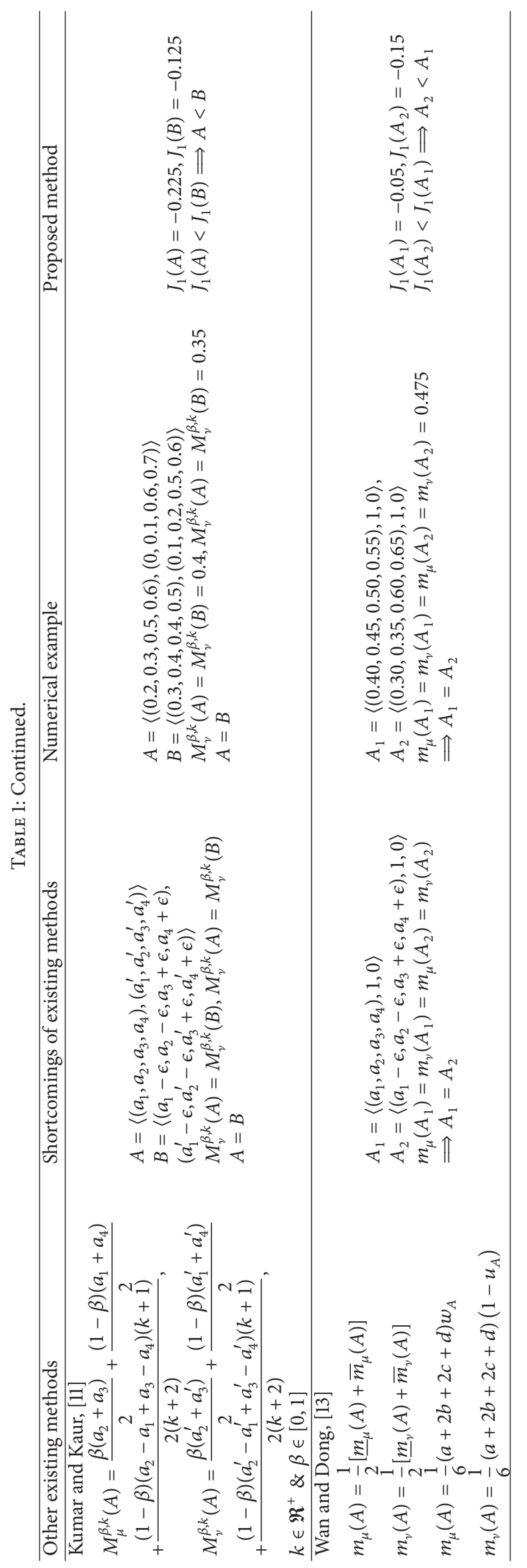




\section{Significance of the Proposed Method}

In this section, significance of our proposed method is shown by comparing our proposed method with all other existing techniques. Table 1 will show the shortcoming of existing methods and the advantage of our method. In Table 1, any trapezoidal intuitionistic fuzzy number is considered as normal.

That is, $A=\left\langle\left(a_{1}, a_{2}, a_{3}, a_{4}\right),\left(a_{1}^{\prime}, a_{2}^{\prime}, a_{3}^{\prime}, a_{4}^{\prime}\right), 1,0\right\rangle$. For example, let $A=\langle(0.2,0.3,0.5,0.6),(0,0.1,0.6,0.7)\rangle$ and $B=\langle(0.3,0.4,0.4,0.5),(0.1,0.2,0.5,0.6)\rangle$ be any two TrIFNs. Then by applying Nehi's [7] approach, we get $C_{\mu}^{k}(A)=$ $C_{\mu}^{k}(B)=0.4$ and $C_{\nu}^{k}(A)=C_{\nu}^{k}(B)=0.35$ and this implies that $A$ and $B$ are equal which is illogical. By applying the total order relation defined in Definition 100, we get $J_{1}(A)=$ $0.225>J_{1}(B)=0.125 \Rightarrow A>B$. Hence our proposed method ranks $A$ as better.

\section{Conclusion}

In this paper, a complete ranking on the class of trapezoidal intuitionistic fuzzy numbers using eight different scores is proposed and compared with existing techniques using illustrative examples. A complete ranking on the class of TrIFNs yields the better results in MADM problems, fuzzy information systems, and artificial intelligence involving intuitionistic trapezoidal fuzzy information and hence our proposed method may be applied to control systems and other engineering fields. Our proposed method is conceptually easy to understand and operationally easy to use.

\section{Competing Interests}

The authors declare that there is no conflict of interests regarding the publication of this paper.

\section{Acknowledgments}

The corresponding author thanks the Council of Scientific and Industrial Research (CSIR-HRDG), India, for supporting this research under CSIR SRF.

\section{References}

[1] R. Jain, "A procedure for multiple-aspect decision making using fuzzy sets," International Journal of Systems Science, vol. 8, no. 1, pp. 1-7, 1977.

[2] D.-F. Li, "A ratio ranking method of triangular intuitionistic fuzzy numbers and its application to MADM problems," Computers \& Mathematics with Applications, vol. 60, no. 6, pp. 15571570, 2010.

[3] G. Deschrijver, "Arithmetic operators in interval-valued fuzzy set theory," Information Sciences, vol. 177, no. 14, pp. 2906-2924, 2007.

[4] A. I. Ban, Intuitionistic Fuzzy Measures. Theory and Applications, Nova Science, New York, NY, USA, 2006.

[5] K. Atanassov, P. Vassilev, and R. Tsvetkov, Intuitionistic Fuzzy Sets, Measures and Integrals, "Prof. M. Drinov" Academic Publishing House, Sofia, Bulgaria, 2013.
[6] H. M. Nehi and H. R. Maleki, "Intuitionistic fuzzy numbers and its applications in fuzzy optimization problem," in Proceedings of the 9th WSEAS CSCC Multiconference Vouliagmeni, Athens, Greece, July 2005.

[7] H. M. Nehi, "A new ranking method for intuitionistic fuzzy numbers," International Journal of Fuzzy Systems, vol. 12, no. 1, pp. 80-86, 2010.

[8] J. Ye, "Expected value method for intuitionistic trapezoidal fuzzy multicriteria decision-making problems," Expert Systems with Applications, vol. 38, no. 9, pp. 11730-11734, 2011.

[9] D. Dubey and A. Mehara, "Linear programming with triangular intuitionistic fuzzy number," in Proceedings of the 7th Conference of the European Society for Fuzzy Logic and Technology, pp. 563569, Aix-les-Bains, France, July 2011.

[10] M. J. Zhang and J. X. Nan, "A compromise ratio ranking method of triangular intuitionistic fuzzy numbers and its application to MADM problems," Iranian Journal of Fuzzy Systems, vol. 10, no. 6, pp. 21-37, 2013.

[11] A. Kumar and M. Kaur, "A ranking approach for intuitionistic fuzzy numbers and its application," Journal of Applied Research and Technology, vol. 11, no. 3, pp. 381-396, 2013.

[12] X.-T. Zeng, D.-F. Li, and G.-F. Yu, "A value and ambiguity-based ranking method of trapezoidal intuitionistic fuzzy numbers and application to decision making," The Scientific World Journal, vol. 2014, Article ID 560582, 8 pages, 2014.

[13] S.-P. Wan and J.-Y. Dong, "Power geometric operators of trapezoidal intuitionistic fuzzy numbers and application to multiattribute group decision making," Applied Soft Computing, vol. 29, pp. 153-168, 2015.

[14] D. Liu, C. Wu, Q. Zhou, and H.-K. Lam, "Fuzzy guaranteed cost output tracking control for fuzzy discrete-time systems with different premise variables," Complexity, vol. 21, no. 5, pp. 265276, 2016.

[15] M. H. Firouz and N. Ghadimi, "Optimal preventive maintenance policy for electric power distribution systems based on the fuzzy AHP methods," Complexity, vol. 21, no. 6, pp. 70-88, 2016.

[16] M. Syed Ali, P. Balasubramaniam, F. A. Rihan, and S. Lakshmanan, "Stability criteria for stochastic Takagi-Sugeno fuzzy Cohen-Grossberg BAM neural networks with mixed timevarying delays," Complexity, vol. 21, no. 5, pp. 143-154, 2016.

[17] P. Liu and F. Teng, "An extended TODIM method for multiple attribute group decision-making based on 2-dimension uncertain linguistic variable," Complexity, vol. 21, no. 5, pp. 20-30, 2016.

[18] P. Liu and F. Teng, "Multiple criteria decision making method based on normal interval-valued intuitionistic fuzzy generalized aggregation operator," Complexity, vol. 21, no. 5, pp. 277290, 2016.

[19] V. Lakshmana Gomathi Nayagam, S. Jeevaraj, and P. Dhanasekaran, "A linear ordering on the class of Trapezoidal intuitionistic fuzzy numbers," Expert Systems with Applications, vol. 60, pp. 269-279, 2016.

[20] V. L. G. Nayagam, S. Jeevaraj, and G. Sivaraman, "Ranking of incomplete trapezoidal information," Soft Computing, 2016.

[21] V. L. G. Nayagam, S. Jeevaraj, and S. Geetha, "Total ordering for intuitionistic fuzzy numbers," Complexity, vol. 21, no. S2, pp. 54-66, 2016. 
[22] V. L. G. Nayagam, S. Jeevaraj, and G. Sivaraman, "Total ordering defined on the set of intuitionistic fuzzy numbers," Journal of Intelligent \& Fuzzy Systems, vol. 30, no. 4, pp. 2015-2028, 2016.

[23] K. T. Atanassov, "Intuitionistic fuzzy sets," Fuzzy Sets and Systems, vol. 20, no. 1, pp. 87-96, 1986.

[24] P. Burillo, H. Bustince, and V. Mohedano, "Some definitions of intuitionistic fuzzy number. First properties," in Proceedings of the 1st Workshop on Fuzzy Based Expert Systems, D. Lakov, Ed., pp. 53-55, Sofia, Bulgaria, September 1994.

[25] K. T. Atanassov and G. Gargov, "Interval valued intuitionistic fuzzy sets," Fuzzy Sets and Systems, vol. 31, no. 3, pp. 343-349, 1989.

[26] P. K. De and D. Das, "Ranking of trapezoidal intuitionistic fuzzy numbers," in Proceedings of the 12th International Conference on Intelligent Systems Design and Applications (ISDA '12), pp. 184188, IEEE, Kochi, India, November 2012. 


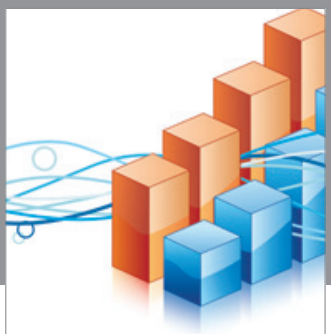

Advances in

Operations Research

vatem alat4

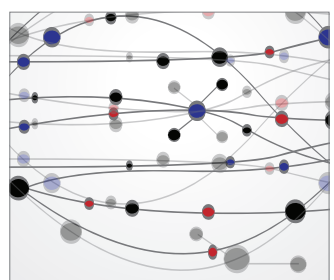

\section{The Scientific} World Journal
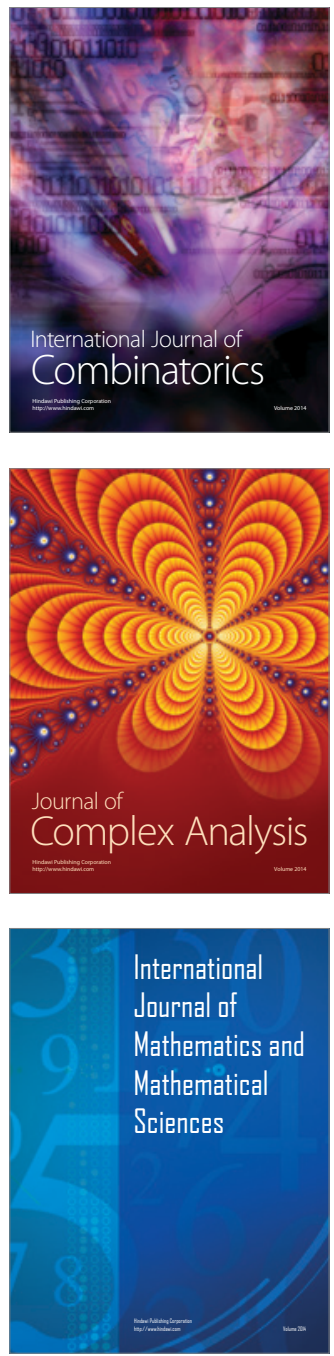
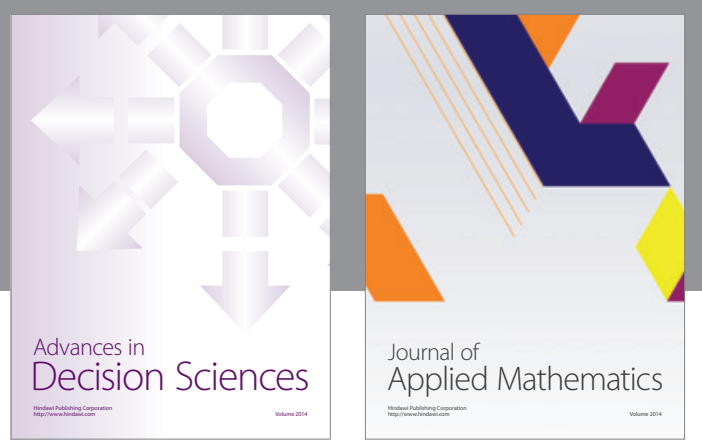

Algebra

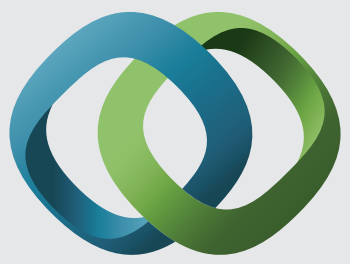

\section{Hindawi}

Submit your manuscripts at

https://www.hindawi.com
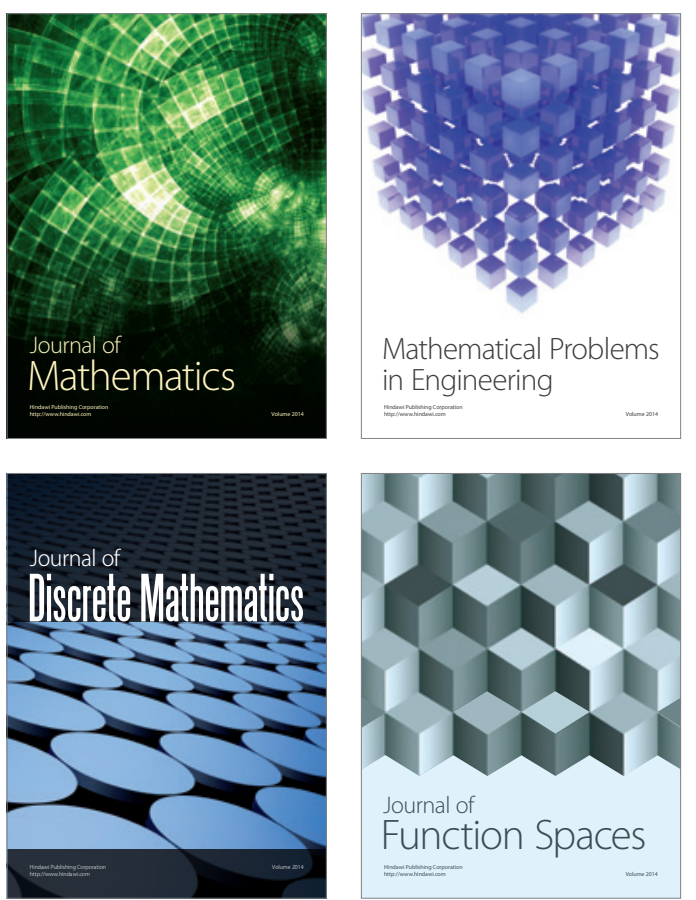

Mathematical Problems in Engineering
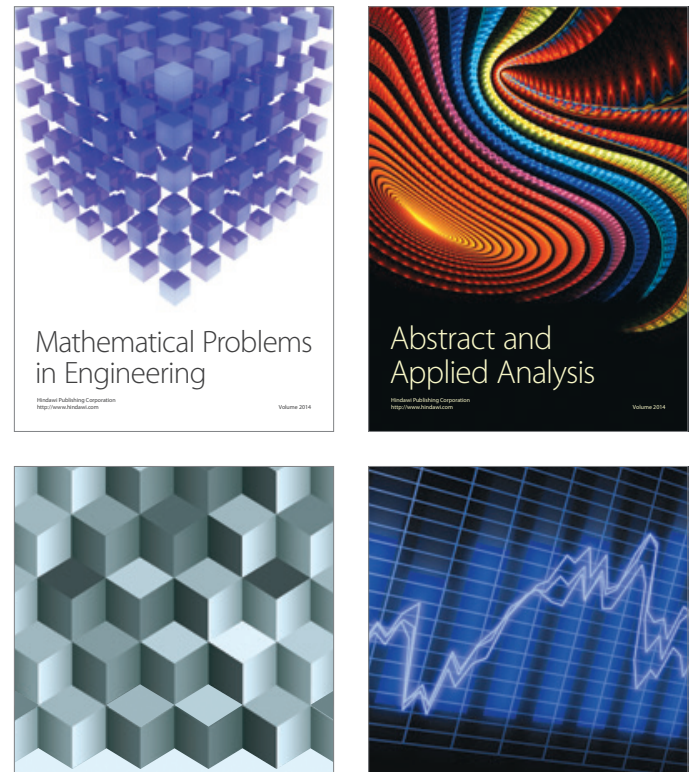

Journal of

Function Spaces

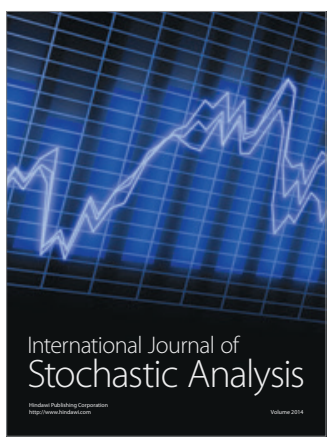

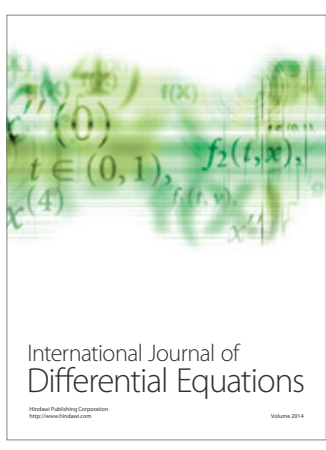
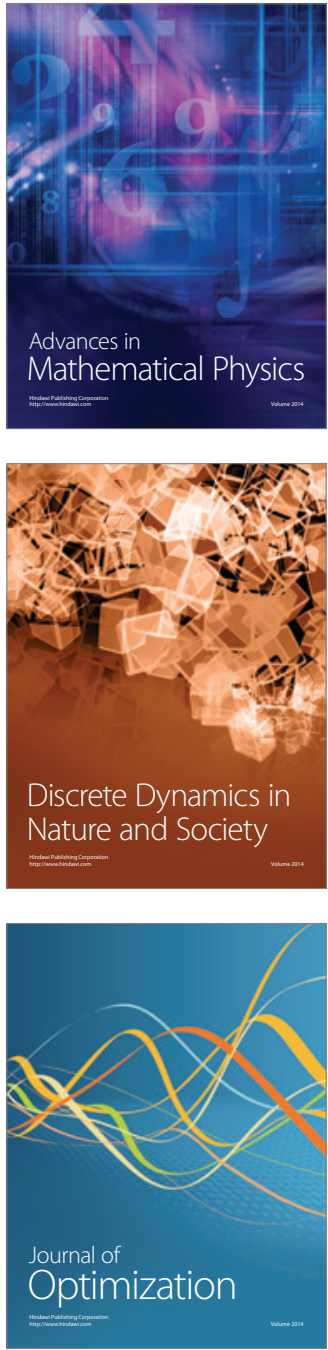